\title{
SPECTROSCOPIC CONFIRMATION OF YOUNG PLANETARY-MASS COMPANIONS ON WIDE ORBITS*
}

\author{
Brendan P. Bowler ${ }^{1,2,4,5}$, Michael C. LiU ${ }^{2}$, Adam L. Kraus ${ }^{3}$, and Andrew W. ManN ${ }^{2,3,6}$ \\ ${ }^{1}$ California Institute of Technology, Division of Geological and Planetary Sciences, \\ 1200 East California Boulevard, Pasadena, CA 91101, USA; bpbowler@caltech.edu \\ 2 Institute for Astronomy, University of Hawai ‘i, 2680 Woodlawn Drive, Honolulu, HI 96822, USA \\ ${ }^{3}$ Astronomy Department, University of Texas at Austin, Austin, TX 78712, USA. \\ Received 2013 November 25; accepted 2014 January 28; published 2014 March 5
}

\begin{abstract}
We present moderate-resolution $(R \sim 4000-5000)$ near-infrared integral field spectroscopy of the young (1-5 Myr) 6-14 $M_{\text {Jup }}$ companions ROXs 42B b and FW Tau b obtained with Keck/OSIRIS and Gemini-North/NIFS. The spectrum of ROXs 42B b exhibits clear signs of low surface gravity common to young L dwarfs, confirming its extreme youth, cool temperature, and low mass. Overall, it closely resembles the free-floating 4-7 $M_{\text {Jup }}$ L-type Taurus member 2MASS J04373705+2331080. The companion to FW Tau AB is more enigmatic. Our optical and near-infrared spectra show strong evidence of outflow activity and disk accretion in the form of line emission from [S II], [O I], $\mathrm{H} \alpha, \mathrm{Ca} I \mathrm{I},[\mathrm{Fe} \mathrm{II}], \mathrm{Pa} \beta$, and $\mathrm{H}_{2}$. The molecular hydrogen emission is spatially resolved as a single lobe that stretches $\approx 0$ !' 1 (15 AU). Although the extended emission is not kinematically resolved in our data, its morphology resembles shock-excited $\mathrm{H}_{2}$ jets primarily seen in young Class 0 and Class I sources. The near-infrared continuum of FW Tau b is mostly flat and lacks the deep absorption features expected for a cool, late-type object. This may be a result of accretion-induced veiling, especially in light of its strong and sustained H $\alpha$ emission $(\mathrm{EW}(\mathrm{H} \alpha) \gtrsim 290 \AA)$. Alternatively, FW Tau b may be a slightly warmer (M5-M8) accreting low-mass star or brown dwarf $\left(0.03-0.15 M_{\odot}\right)$ with an edge-on disk. Regardless, its young evolutionary stage is in stark contrast to its Class III host FW Tau AB, indicating a more rapid disk clearing timescale for the host binary system than for its wide companion. Finally, we present near-infrared spectra of the young ( 2-10 Myr) low-mass $\left(12-15 M_{\text {Jup }}\right)$ companions GSC 6214-210 B and SR 12 C and find they best resemble low-gravity M9.5 and M9 substellar templates.
\end{abstract}

Key words: brown dwarfs - planetary systems - stars: individual (ROXs 42B, FW Tau, GSC 6214-210, SR 12) stars: low-mass

Online-only material: color figures

\section{INTRODUCTION}

Adaptive optics (AO) imaging searches for self-luminous giant planets are uncovering a population of companions with separations of several hundred AU and masses near and below the deuterium-burning limit ( $\sim 13 M_{\text {Jup }}$; e.g., Spiegel et al. 2011). Together with directly imaged planets located at more moderate separations of 10-100 AU (Marois et al. 2008, 2010; Lagrange et al. 2010; Kraus \& Ireland 2012; Kuzuhara et al. 2013; Rameau et al. 2013), planetary-mass companions (PMCs) on wide orbits are extending the scale of planetary systems to over five orders of magnitude in separation $(\sim 0.1-1000 \mathrm{AU})$. Altogether, a total of 12 PMCs to stars with projected separations in excess of $100 \mathrm{AU}$ and masses $\lesssim 15 M_{\text {Jup }}$ have been found (Table 1). With the exception of Ross $458 \mathrm{C}$ and WD 0806-661 $\mathrm{B}$, all of these systems are very young (most being $\lesssim 10 \mathrm{Myr}$ ) and the companion spectral types span late-M to mid-L. This selection for extreme youth is primarily a result of brown dwarf and giant planet evolution; by $1 \mathrm{Gyr}, 10 M_{\mathrm{Jup}}$ objects cool to a

\footnotetext{
* Some of the data presented herein were obtained at the W. M. Keck Observatory, which is operated as a scientific partnership among the California Institute of Technology, the University of California and the National Aeronautics and Space Administration. The Observatory was made possible by the generous financial support of the W. M. Keck Foundation.

4 Visiting Astronomer at the Infrared Telescope Facility, which is operated by the University of Hawaii under Cooperative Agreement no. NNX-08AE38A with the National Aeronautics and Space Administration, Science Mission Directorate, Planetary Astronomy Program.

5 Caltech Joint Center for Planetary Astronomy Fellow.

6 Harlan J. Smith Fellow.
}

mere 500 K (Burrows et al. 1997; Baraffe et al. 2003), placing them at the boundary between $\mathrm{T}$ and Y dwarfs (Cushing et al. 2011) where near-infrared (NIR) absolute magnitudes plummet (Kirkpatrick et al. 2011, 2012; Liu et al. 2011, 2012). At $5 \mathrm{Myr}$, however, the same objects have effective temperatures of $\sim 2000 \mathrm{~K}$ and absolute magnitudes of $\sim 10-11 \mathrm{mag}$ in the NIR assuming "hot start" formation (Marley et al. 2007), making them easy targets for NIR AO imaging surveys of star-forming regions (e.g., Ireland et al. 2011).

The existence of these wide-separation PMCs poses a challenge for models of giant planet formation. Their orbital separations are far too large for core accretion to operate (e.g., Pollack et al. 1996; Alibert et al. 2005; Dodson-Robinson et al. 2009) and models of disk instability are most efficient at smaller separations of tens of AU where protoplanetary disks are both massive and cold enough to gravitationally collapse (Boss 1997, 2011; Mayer 2002; Kratter et al. 2010). Other formation scenarios include the direct fragmentation of molecular cloud cores (e.g., Bate et al. 2002; Bate 2012) and planet-planet scattering to wide orbits (Boss 2006; Scharf \& Menou 2009; Veras et al. 2009). Deciphering which mechanism dominates requires testable predictions from theory and accurate demographics for PMCs, neither of which is currently available.

Observationally, the statistics of wide-separation PMCs are only beginning to be measured. At projected separations of 400-4000 AU, Aller et al. (2013) infer a frequency of $0.6 \pm 0.3 \%$ for substellar companions with masses of 15-60 $M_{\text {Jup }}$, which implies a frequency of $<0.6 \%$ for companions with masses of $\sim 5-15 M_{\text {Jup }}$ based on their sensitivity limits ( $\sim M_{\text {Jup }}$ ). 
Table 1

Wide (>100 AU) Companions Under $15 M_{\text {Jup }}$ Around Stars

\begin{tabular}{|c|c|c|c|c|c|c|c|c|}
\hline Object & $\begin{array}{c}\text { Mass } \\
\left(M_{\text {Jup }}\right)\end{array}$ & $\begin{array}{l}\text { Age } \\
(\mathrm{Myr})\end{array}$ & $\begin{array}{c}\text { Separation } \\
(\mathrm{AU})\end{array}$ & NIR SpT & Pri. Multiplicity ${ }^{\mathrm{a}}$ & $\begin{array}{l}\text { Pri. Mass } \\
\qquad\left(M_{\odot}\right)\end{array}$ & Pri. SpT & References \\
\hline WD 0806-661 B & $6-9$ & $2000 \pm 500$ & 2500 & Y? & $\mathrm{S}$ & $2.0 \pm 0.3$ & $\mathrm{DQ}^{\mathrm{b}}$ & $1,2,3$ \\
\hline HD 106906 b & $11 \pm 2$ & $13 \pm 2$ & $\sim 650$ & $\mathrm{~L} 2.5 \pm 1$ & S & $1.50 \pm 0.10$ & F5 & 4,5 \\
\hline 1RXS J1609-2105 b & $8_{-2}^{+4}$ & $\sim 5$ & $\sim 330$ & $\mathrm{~L} 4 \pm 1$ & S & $0.85_{-0.10}^{+0.20}$ & $\mathrm{M} 0 \pm 1$ & $6,7,8,9$ \\
\hline ROXs 42B b & $6-14$ & $7_{-2}^{+3}$ & $\sim 140$ & $\mathrm{~L} 1 \pm 1$ & $\mathrm{~B}$ & $0.89 \pm 0.08,0.36 \pm 0.04$ & $\mathrm{M} 1 \pm 1$ & 9,10 \\
\hline FW Tau b & $6-14$ & $2_{-0.5}^{+1}$ & $\sim 330$ & pec & $\mathrm{B}$ & $0.28 \pm 0.05,0.28 \pm 0.05$ & $\mathrm{M} 6 \pm 1$ & 9,10 \\
\hline Ross $458 \mathrm{C}$ & $6-12$ & $150-800$ & 1190 & $\mathrm{~T} 8.5 \mathrm{p} \pm 0.5$ & B & $\approx 0.6, \approx 0.09$ & $\mathrm{M} 0.5 \pm 0.5, \sim \mathrm{M} 7$ & $11,12,13,14,15,16$ \\
\hline ROXs 12 b & $12-20$ & $8_{-3}^{+4}$ & $\sim 210$ & $\ldots$ & $\mathrm{S}$ & $0.87 \pm 0.08$ & $\mathrm{M} 0 \pm 1$ & 10,17 \\
\hline AB Pic B & $13-14$ & $\sim 30$ & 250 & $\mathrm{~L} 0 \pm 1$ & S & $0.95 \pm 0.05^{\mathrm{c}}$ & $\mathrm{K} 2$ & 18,19 \\
\hline CHXR 73 B & $13_{-6}^{+8}$ & $\sim 2$ & $\sim 210$ & $\geqslant \mathrm{M} 9.5$ & S & 0.30 & $\mathrm{M} 3.25 \pm 0.25$ & 20,21 \\
\hline DH Tau B & $12_{-4}^{+10}$ & $\sim 1-2$ & $\sim 340$ & M9.25 \pm 0.25 & S & 0.53 & M2 & $17,22,23,24$ \\
\hline GSC 6214-210 B & $14 \pm 2$ & $\sim 5$ & $\sim 320$ & M9.5 \pm 1 & $\mathrm{~S}$ & $0.9 \pm 0.1$ & $\mathrm{~K} 5 \pm 1$ & $8,9,25$ \\
\hline $\mathrm{SR} 12 \mathrm{C}$ & $12-15$ & $\sim 2$ & $\sim 1100$ & $\mathrm{M} 9.0 \pm 0.5$ & $\mathrm{~B}$ & $1.05 \pm 0.05,0.5 \pm 0.1^{\mathrm{d}}$ & $\mathrm{K} 4 \pm 1, \mathrm{M} 2.5 \pm 1$ & 9,26 \\
\hline
\end{tabular}

Notes.

a Single ("S") or binary ("B").

b WD 0806-661 is a DQ white dwarf.

${ }^{c}$ Based on evolutionary models from Baraffe et al. (1998) using the age (30 Myr, Song et al. 2003) and luminosity (0.5 $L_{\odot}$; McDonald et al. 2012) of AB Pic.

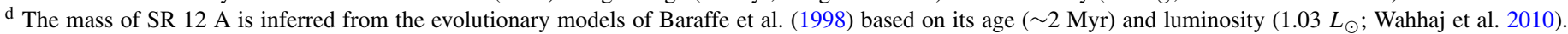

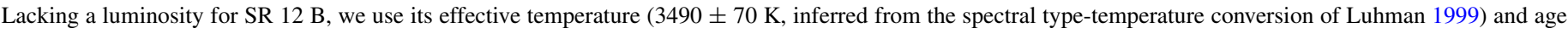
to compute a mass from evolutionary models.

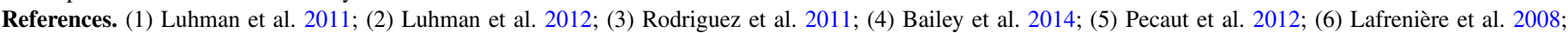

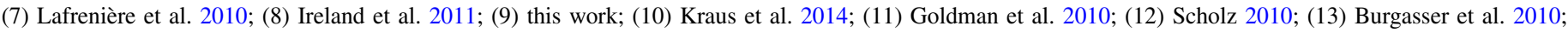

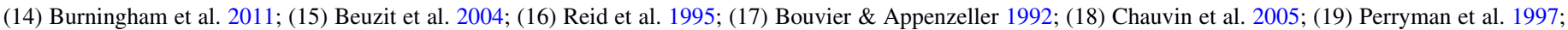

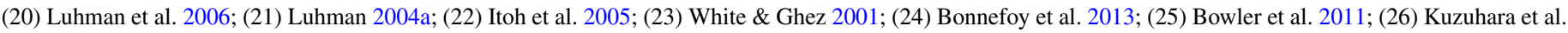
2011.

This compares to an occurrence rate of $4_{-1}^{+5} \%$ by Ireland et al. (2011) for 6-20 Mup companions between 200-500 AU. Upper limits from high-contrast imaging surveys are yielding consistent results. For example, Nielsen \& Close (2010) found that fewer than $20 \%$ of solar-mass stars harbor $>4 M_{\text {Jup }}$ planets between $\approx 20$ and $500 \mathrm{AU}$ at the $95 \%$ confidence level. At smaller separations of 10-150 AU, fewer than 6\% of stars harbor 1-20 $M_{\text {Jup }}$ planets (Biller et al. 2013). PMCs therefore appear to be more common at several hundred AU than several thousand $\mathrm{AU}$, but larger samples are needed to make any strong conclusions about their separation distributions.

Regardless of their origin, wide-separation PMCs are valuable for studying the atmospheres of young gas giants. Their relatively large angular separations (typically $\sim 1-3^{\prime \prime}$ ) and modest contrasts ( 5-7 mag in $H$-band) make them more accessible to spectroscopic observations than the current population of "bona fide" directly imaged planets, which generally reside at smaller separations and have less favorable contrasts (>10 mag). Follow-up near-infrared spectroscopy of several PMCs has confirmed their low temperatures and youth (e.g., Lafrenière et al. 2008; Bonnefoy et al. 2010; Bowler et al. 2011; Patience et al. 2012). Overall, they exhibit several low-gravity features first identified in their young brown dwarf counterparts in star-forming regions (e.g., Lucas et al. 2001; Slesnick et al. 2004; Allers et al. 2007; Bonnefoy et al. 2013) and the field (McGovern et al. 2004; Kirkpatrick et al. 2006; Allers \& Liu 2013), most notably angular $H$-band shapes and shallow $J$-band alkali line strengths. Eventually, the near-IR spectra of wide PMCs can be used to map the influence of effective temperature and surface gravity (or equivalently, mass and age) on spectral morphology in order to calibrate more challenging spectroscopic studies of giant planets at smaller angular separations (Bowler et al. 2010; Barman et al. 2011; Oppenheimer et al. 2013; Konopacky et al. 2013).
Kraus et al. (2014) have recently discovered three new PMCs from follow-up imaging of candidate planetary companions reported in the literature. Initially identified in binary surveys of the Taurus and $\rho$ Ophiucus star-forming regions (White \& Ghez 2001; Ratzka et al. 2005), these PMCs have been shown to be comoving with their host stars at projected separations of hundreds of AU. Here we present follow-up NIR spectroscopy for two of these new companions, FW Tau b and ROXs 42B b. FW Tau b orbits the tight stellar binary FW Tau AB at a projected separation of $330 \mathrm{AU}\left(2^{\prime \prime} .3\right)$. A member of the Taurus starforming region, the system has an age of $\sim 2 \mathrm{Myr}$, leading to a model-dependent mass of 5-13 $M_{\text {Jup }}$ for the companion. ROXs $42 \mathrm{~B}$ b has a similar mass estimate of 5-12 $M_{\text {Jup }}$ and is bound to the tight $\rho$ Oph binary ROXs $42 \mathrm{~B}$ AB at $140 \mathrm{AU}\left(1^{\prime \prime} .2\right)$ separation (see also Currie et al. 2014). The age estimate for the system is $\sim 7 \mathrm{Myr}$ based on the HR diagram positions of the stellar binary components (Kraus et al. 2014). Here we present spectroscopic follow-up observations of these companions in an effort to confirm their cool temperatures and low masses. Together these objects are among the lowest-mass directly imaged companions known.

In addition, we present NIR spectroscopy of the PMCs GSC 6214-210 B and SR12 C (a.k.a., 2MASS J16271951-2441403 C). GSC $6214-210 \mathrm{~B}$ is a $\sim 14 M_{\text {Jup }}$ companion discovered by Ireland et al. (2011) in a shallow imaging search for PMCs in Upper Scorpius complex (5-10 Myr). Bowler et al. (2011) presented $J$ - and $H$-band spectroscopy of GSC 6214$210 \mathrm{~B}$ which revealed a spectral type of $\mathrm{LO}_{-1}^{+2}$. Notably, they found extraordinarily strong $\mathrm{Pa} \beta$ emission originating from the companion, indicating vigorous accretion from a circumsubstellar disk. Here we present a $K$-band spectrum of GSC 6214-210 B. Finally, we have also obtained a NIR spectrum of the 12-15 $M_{\text {Jup }}$ PMC SR12 C, discovered by Kuzuhara et al. (2011) as part of a search for substellar companions to members 
of the $\rho$ Oph complex. With a separation of $\sim 1100$ AU, it is among the widest-separation PMCs known. Kuzuhara et al. presented low-resolution NIR spectroscopy of this object; here we present the first moderate-resolution NIR spectrum.

\section{OBSERVATIONS}

\subsection{Keck/OSIRIS NIR Spectroscopy of FW Tau b, ROXs $42 B$ b, and GSC 6214-210 B}

We observed FW Tau b with the OH-Suppressing Infrared Imaging Spectrograph (OSIRIS; Larkin et al. 2006) on 2011 October 4 UT with the Keck II $10 \mathrm{~m}$ telescope using natural guide star adaptive optics (NGSAO). The 50 mas plate scale was used for both the $H b b(1.473-1.803 \mu \mathrm{m})$ and $K b b$ $(1.965-2.381 \mu \mathrm{m})$ filters, resulting in a $0.2 \times 3.2$ field of view and a resolving power $(R \equiv \lambda / \delta \lambda)$ of $\sim 3800$. The long axis was oriented parallel to the star-companion position angle (P.A.) and the telescope was nodded along the detector by $1^{\prime \prime}$ in an ABBA pattern. Sky frames were taken before and after the observations. Seeing measurements from the DIMM on Canada-France-Hawaii Telescope (CFHT) were between $\approx 0$.'8-1".0 during the observations and modest levels of cirrus were present throughout the night. We obtained six $300 \mathrm{~s}$ exposures in $H b b$ and $13300 \mathrm{~s}$ exposures in $K b b$ at an airmass of 1.01-1.04. Immediately after our science observations we observed the A0V star HD 35036 at a similar airmass to correct for telluric absorption.

In early 2012 OSIRIS moved to the Keck I telescope, and on 2013 February 1 and 3 UT we obtained additional NIR spectroscopy of FW Tau b with Keck I/OSIRIS. Our February 1 observations were carried out with laser guide star AO using FW Tau AB as the on-axis tip-tilt star. The wavefront sensor (WFS) $R$-band magnitude of the laser was $\sim 9.7-10.2$ mag. Natural seeing was poor, with CFHT DIMM reporting $\approx 1^{\prime \prime} .2$. We obtained a total of 100 minutes of integration time in $J b b$ and 60 minutes in $K b b$ by nodding along the detector, which was oriented perpendicular to the star-companion axis. On February 3 we obtained a second epoch of $H b b$ data in NGSAO mode. The WFS $R$-band magnitude of FW Tau AB was $14.0 \mathrm{mag}$, and seeing varied between $\approx 0.5-11^{\prime \prime} .0$. The observations were carried out in the same fashion as the other bands, and we acquired a total of 30 minutes of integration time on the science target. The same A0V standard, HD 35036, was used on both nights.

We obtained a $K$-band spectrum of ROXs 42B b on 2011 August 20 UT with OSIRIS on Keck II using NGSAO. CFHTDIMM reported seeing measurements of $\approx 0^{\prime \prime} 6$ during the observations. In addition to the bona fide comoving companion ROXs 42B b, Kraus et al. (2014) also identified a candidate companion located interior to ROXs $42 \mathrm{~B}$ b at a mere 0.55 from the primary star, but astrometry revealed that this inner candidate is likely a background star. At the time of our OSIRIS observations, the status of the inner object was more uncertain, so we attempted to obtain spectra of both of these companions simultaneously. We chose the 35 mas plate scale to compensate between finely sampling the speckles at small separations and obtaining a sufficiently large field of view $(0.56 \times 2$ '.24) to encompass both objects. The orientation of the detector was parallel to the inner-companion-outer-companion P.A., and we nodded along the detector axis in an ABBA pattern by 0.'4. We obtained nine $300 \mathrm{~s}$ exposures with the $K b b$ filter at an airmass range of 1.43-1.57. Prior to our science observations we observed the A0V standard HD 157734 at an airmass of 1.33 for telluric correction.

On 2012 June UT we observed GSC 6214-210 B with OSIRIS on Keck I using NGSAO. The long axis of the detector was oriented perpendicular to the GSC 6214-210 A-B axis and was nodded in an ABBA fashion by $1^{\prime \prime}$. We obtained eight $300 \mathrm{~s}$ exposures in $K b b$ using the 50 mas plate scale. The airmass range of our science observations was 1.36-1.46. We then observed the A0V telluric standard HD 157170 at an airmass of 1.3.

Our OSIRIS observations of FW Tau b, ROXs 42B b, and GSC 6214-210 B were reduced in the same manner. The OSIRIS Data Reduction Pipeline was used for basic image reduction, which includes sky subtraction to remove night-sky emission lines, corrections to account for DC bias shifts and electronic crosstalk among the output channels, the removal of cosmic rays and bad pixels, and rectification of the two-dimensional (2D) spectra into three-dimensional (3D) data cubes. The rectification matrices measured closest in time prior to the observation dates were used for this last step. Sky subtraction was performed with sky frames obtained after the science observations for our $2011 \mathrm{FW}$ Tau b data, whereas consecutive ABBA nodded frames were used for ROXs 42B b, GSC 6214-210 B, and our 2013 FW Tau b data.

The science and standard spectra were extracted from the rectified data cubes using aperture photometry. Aperture radii of 3 spaxels were centered on the median-combined centroid position of the targets. For our science frames we implemented sky subtraction measured from a large annulus surrounding the target. Because the observations of the ROXs 42B companion were nodded by only 0.4 , we used a smaller aperture radius of 2.5 spaxels without sky subtraction to avoid the negative dipole from the pairwise-subtracted nods. Finally, telluric correction was performed for all our OSIRIS data using the xtellcor_general routine from the Spextool IRTF data reduction package (Cushing et al. 2004) based on the method described in Vacca et al. (2003).

The inner point source located at 0.55 from ROXs $42 \mathrm{~B}$ is visible in our data and is clearly distinguishable from nearby point spread function (PSF) speckles because of the wavelengthdependent PSF structure. However this inner candidate is embedded in the wing of the star's PSF, and there are several nearby bright speckles which make the extraction non-trivial. We attempted to use spectral deconvolution with masking (Thatte et al. 2007), but because of the close proximity of nearby speckles, the result is unreliable.

\subsection{Gemini-North/NIFS NIR Spectroscopy of ROXs $42 B b$}

We obtained $J$ - and $H$-band spectroscopy of ROXs $42 \mathrm{~B}$ b with the Near-Infrared Integral Field Spectrometer (NIFS; McGregor et al. 2003) on the Gemini-North $8.1 \mathrm{~m}$ telescope in combination with the facility AO system ALTAIR (Herriot et al. 1998). NIFS is an image-slicer integral field spectrograph with $0^{\prime \prime} .10 \times$ 0. .04 rectangular spaxels, providing imaging and $R \sim 5000$ spectroscopy over a 3 . $0 \times 3$ 3. 0 field of view.

Our $H$-band observations of ROXs $42 \mathrm{~B}$ b were obtained on 2012 May 9 UT. $J$-band data were taken at two different dates, 2012 May 14 UT and 2012 July 4 UT, due to scheduling constraints. The observations were carried out in queue mode (Program ID: GN-2012A-Q-48) using NGSAO with the primary star acting as the natural guide star $(R=13.4 \mathrm{mag}$; Zacharias et al. 2013). The Cassegrain rotator was set to track sidereal motion. To avoid saturating the detector, we placed the primary off the array by 0.70 (the primary-companion separation is $1^{\prime \prime} .17$ ) 

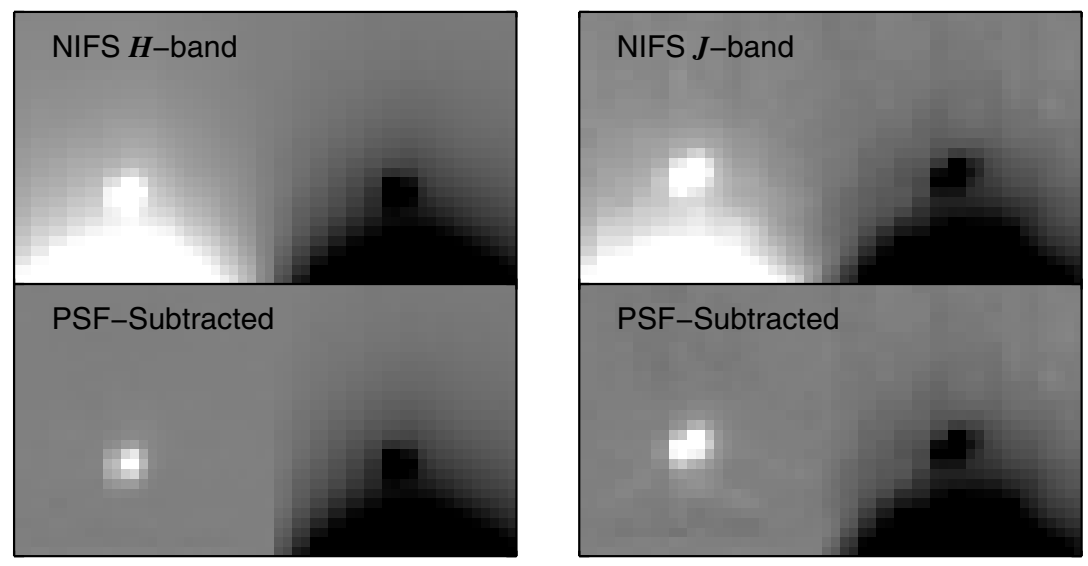

Figure 1. Gemini-N/NIFS observations of ROXs 42B b. The upper images show examples of collapsed, pair-subtracted $H$-and $J$-band data cubes. ROXs $42 \mathrm{~B}$ b is clearly visible but appears in the wing of the primary star's PSF (the separation is 1 .'17). The bottom panels show the same pair-subtracted images after fitting and subtracting a Moffat function to the contaminating flux for the positive image.

Table 2

Spectroscopic Observations

\begin{tabular}{|c|c|c|c|c|c|c|}
\hline Object & $\begin{array}{l}\text { Date } \\
\text { (UT) }\end{array}$ & $\begin{array}{l}\text { Telescope/ } \\
\text { Instrument }\end{array}$ & Filter & $\begin{array}{l}\text { Plate Scale } \\
\text { (mas) }\end{array}$ & $\begin{array}{l}\text { Exp. Time } \\
\text { (minute) }\end{array}$ & Standard \\
\hline FW Tau b & 2011 Oct 4 & Keck-II/OSIRIS & $H b b$ & 50 & 30 & HD 35036 \\
\hline FW Tau b & 2011 Oct 4 & Keck-II/OSIRIS & $K b b$ & 50 & 65 & HD 35036 \\
\hline FW Tau b & 2013 Feb 1 & Keck-I/OSIRIS & $J b b$ & 50 & 100 & HD 35036 \\
\hline FW Tau b & 2013 Feb 1 & Keck-I/OSIRIS & $K b b$ & 50 & 60 & HD 35036 \\
\hline FW Tau b & 2013 Feb 3 & Keck-I/OSIRIS & $H b b$ & 50 & 30 & HD 35036 \\
\hline FW Tau AB+b & 2011 Oct 8 & UH $2.2 \mathrm{~m} / \mathrm{SNIFS}$ & $\ldots$ & 400 & 15 & HR 718, HD 93521 \\
\hline FW Tau AB+b & 2013 Jan 2 & UH $2.2 \mathrm{~m} / \mathrm{SNIFS}$ & $\ldots$ & 400 & 60 & HR 718, HD 93521 \\
\hline ROXs 42B b & 2012 May 9 & Gemini-N/NIFS & $H^{\mathrm{a}}$ & $100 \times 40$ & 135 & HD 155379 \\
\hline ROXs 42B b & 2012 May 14 & Gemini-N/NIFS & $J^{\mathrm{b}}$ & $100 \times 40$ & 55 & HD 155379 \\
\hline ROXs 42B b & $2012 \mathrm{Jul} 4$ & Gemini-N/NIFS & $J^{\mathrm{b}}$ & $100 \times 40$ & 140 & HD 155379 \\
\hline ROXs 42B b & 2011 Aug 20 & Keck-II/OSIRIS & $K b b$ & 35 & 45 & HD 157734 \\
\hline ROXs 42B AB+b & 2012 May 20 & UH $2.2 \mathrm{~m} / \mathrm{SNIFS}$ & $\ldots$ & 400 & 4 & EG131, HR 5501 \\
\hline 1RXS J160929.1-210524 A & 2012 May 20 & UH $2.2 \mathrm{~m} / \mathrm{SNIFS}$ & $\ldots$ & 400 & 2.5 & EG131, HR 5501 \\
\hline GSC $6214-210$ B & 2012 Jun 26 & Keck-I/OSIRIS & $K b b$ & 50 & 40 & HD 157170 \\
\hline SR12 C & 2012 Jul 11 & IRTF/SpeX-SXD ${ }^{c}$ & $\ldots$ & 150 & 80 & HD 145127 \\
\hline $2 \mathrm{M} 0437+2331$ & 2012 Jan 22 & IRTF/SpeX-prism ${ }^{c}$ & $\ldots$ & 150 & 20 & HD 27761 \\
\hline
\end{tabular}

Notes.

a NIFS $J H$ filter with $H$-band grating.

b NIFS $Z J$ filter with $J$-band grating.

c A slit width of 0. . 8 was used.

and oriented the instrument so that the short axis of the spaxels were aligned with the primary-companion P.A. in order to better sample the primary star's PSF (Figure 1). We obtained pairs of image cubes executed in an ABBA pattern with 1".5 nods while reading the detector in "Medium Object" mode for our science observations. Our $H$-band data ( $H$ grating with the $J H$ filter) consist of $27300 \mathrm{~s}$ exposures (135 minutes total) over an airmass range of 1.44-2.61; although this change in airmass is substantial, only eight frames were acquired above an airmass of 2.0. The 2012 May $J$-band data ( $J$ grating with the $Z J$ filter) consist of $11300 \mathrm{~s}$ exposures (55 minutes total) over an airmass range of 1.49-1.73. Our 2012 July sequence is longer and was better centered during transit; $28300 \mathrm{~s}$ exposures (140 minutes total) were acquired over an airmass range of 1.40-1.73. After each science sequence a set of argon lamp frames were acquired at the same sky position for wavelength calibration. Standard A0V stars were observed in a dithered sequence immediately prior to or after the science sequences (Table 2). The airmasses of the standards are 2.2 for the 2012 May 9 data set, 1.6 for 2012 May 14, and 1.5 for 2012 July 4. Flat fields, NIFS "Ronchi" calibration flats, and dark frames were taken at the end of the night.

Basic data reduction was carried out with the Gemini/ NIFS processing package written for the Image Reduction and Analysis Facility. This includes cleaning the data of cosmic rays and bad pixels, deriving a wavelength solution using the arc lamp frames, pairwise subtracting nodded frames to remove sky features, and rectifying the $2 \mathrm{D}$ spectra into $3 \mathrm{D}$ image cubes. We modified the default NIFS scripts to assemble individual cubes for both the science and standard data without correcting them for telluric absorption. ROXs $42 \mathrm{~B}$ b is clearly visible in the wing of the primary star's PSF (Figure 1, top panel). To remove this contaminating flux, we fit the extended PSF halo with several analytic models and subtracted them from the data while masking out the companion. We used the curve fitting package MPFIT (Markwardt 2009) to separately test three functions - a Gaussian, Lorenztian, and Moffat (Moffat 1969)-by fitting and subtracting them from each column at every wavelength channel for all of our $J$ - and $H$-band science observations (Figure 1, bottom panel). The spectra 


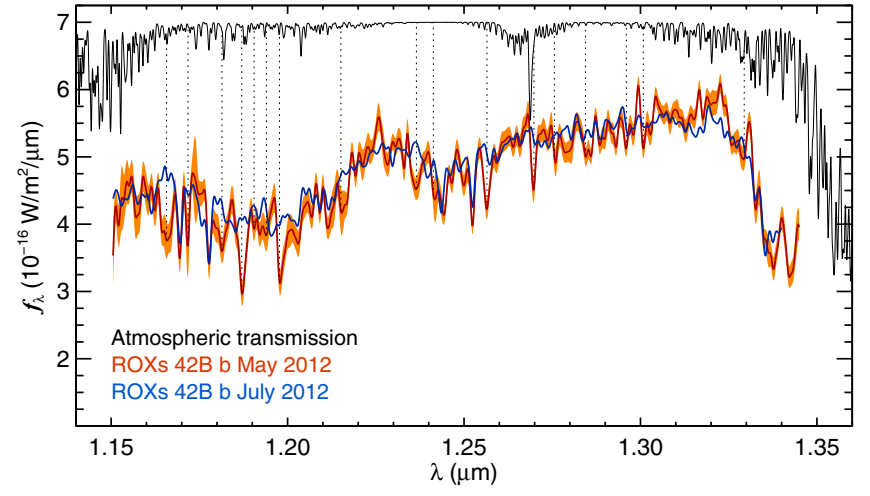

Figure 2. Comparison of our telluric-corrected NIFS $J$-band spectra of ROXs 42B b taken in 2012 May (red) and 2012 July (blue). The overall shape of the spectra agree but the detailed features differ. Several of these discrepant lines (dotted black) in our lower-S/N spectrum from May appear to be of telluric origin (solid black) and are probably caused by poor sky subtraction. We therefore adopt the higher S/N July spectrum for this work. The spectra of ROXs 42B b have been smoothed to a resolving power of 1000 and flux calibrated to the measured $J$-band magnitude $(16.99 \pm 0.07 \mathrm{mag}$; Kraus et al. 2014). $1 \sigma$ standard errors are shown as shaded orange and light blue. The transmission spectrum was generated by ATRAN (Lord 1992) assuming an airmass of 1.5 and a water vapor column of $1.6 \mathrm{~mm}$ (made available by Gemini Observatory).

(A color version of this figure is available in the online journal.)

were then extracted with aperture radii of 3 spaxels $\left(0{ }^{\prime} \cdot 12\right)$. The resulting spectra are in agreement for the three different representations of the extended PSF, indicating that systematics from the subtraction process are minimal. Telluric correction was performed with the xtellcor_general routine in the IRTF/Spextool data reduction package. In Figure 2 we compare our telluric-corrected NIFS $J$-band spectra of ROXs 42B b from 2012 May and July. The lower S/N spectrum from May contains many lines that appear to be telluric in origin, and are probably a result of poor sky subtraction of the data. We therefore adopt the higher S/N July spectrum for this work, although we note that the overall shape of the extracted spectra are very similar at both dates.

\subsection{IRTF/SpeX NIR Spectroscopy of SR 12 C and $2 M A S S 0437+2331$}

We observed SR12 C with IRTF/SpeX in short crossdispersed (SXD) mode on 2012 July 11 UT. The slit was set to 0 ' 8 , resulting in a spectral resolution of $\sim 750$. We obtained 16 nodded pairs of SR12 C (32 individual frames) taken in an ABBA pattern at an airmass of $\approx 1.4$. Individual exposure times were $150 \mathrm{~s}$, providing a total on-source time of 80 minutes. Light cirrus was present throughout the night, and the DIMM mounted on CFHT reported seeing measurements of $0.6-0$.'7 during the observations. We observed the A0V star HD 145127 at a similar airmass for telluric correction. The data were reduced with the IRTF Spextool data reduction pipeline.

As a comparison template for our spectra of ROXs $42 \mathrm{~B} \mathrm{~b}$ and FW Tau b, we also targeted the latest-type member of the Taurus star-forming region currently known, 2MASS J04373705+2331080 (herinafter 2M0437+2331; Luhman et al. 2009), with IRTF/SpeX. The L0 optical spectrum obtained by Luhman et al. revealed strong $\mathrm{H} \alpha$ emission and spectroscopic indications of low surface gravity, confirming the object's youth. At the extremely young age of Taurus, the inferred mass of $2 \mathrm{M} 0437+2331$ is $\sim 4-7 M_{\text {Jup }}$. Our observations of $2 \mathrm{M} 0437+2331$ were made on 2012 January 22 UT in prism mode using a 0.8 slit width $(R \sim 90)$. We acquired a total of
20 minutes $(10 \times 120 \mathrm{~s})$ of data while nodding the telescope along the slit. Immediately afterward we targeted the A0V standard HD 27761. The data were reduced and extracted using Spextool.

\subsection{UH $2.2 \mathrm{~m} /$ SNIFS Optical Spectroscopy of PMC Host Stars and FW Tau b}

In addition to spectroscopy of new PMCs, we are also gathering a uniform set of optical spectra of their primary stars with the University of Hawai'i 2.2 m telescope's SuperNova Integral Field Spectrograph (SNIFS; Lantz et al. 2004) to characterize the host stars in a homogeneous manner (e.g., Bowler et al. 2011). SNIFS is an integral field unit with a resolving power of $\sim 1300$ that simultaneously samples blue (3000-5200 $\AA$ ) and red (5200-9500) spectral regions. We observed ROXs 42B AB and FW Tau AB on 2012 May 20 UT and 2011 October 8 UT, respectively. ROXs $42 \mathrm{~B} \mathrm{AB}$ was targeted for 4 minutes at an airmass of 1.4. with outstanding seeing $\left(\sim 00^{\prime} .5\right)$ as reported by the DIMM mounted on CFHT. We acquired 15 minutes of data for FW Tau AB at an airmass of 1.02. Light cirrus was present throughout the night and seeing was poor $\left(\sim 1^{\prime \prime}\right)$. The O/B-type stars HR 718 and HD 93521 were used as standards.

Similarly, we targeted the PMC host star 1RXS J160929.1210524 A with SNIFS on 2012 May 20 UT. We obtained $147 \mathrm{~s}$ of integration time at an airmass of 1.3. Seeing ranged from $0 . ' 4$ to $1^{\prime \prime}$.0 throughout the night. Note, however, that dome seeing at the UH $2.2 \mathrm{~m}$ telescope limits the angular resolution of our SNIFS observations to $\gtrsim 0$.'8. The white dwarf EG131 and B-type star HR 5501 were used as spectrophotometric standards.

Details about our SNIFS data reduction procedures can be found in Mann et al. (2012). To summarize, the data were processed with the SNIFS data reduction pipeline (Aldering et al. 2006; Scalzo et al. 2010), which includes flat, bias, and dark corrections, rectification of the data into spectral cubes, wavelength calibration using arc lamps, cleaning the data of bad pixels and cosmic rays, and spectral extraction with a PSF model.

Upon close inspection of our SNIFS observation of FW Tau AB, we found that the companion FW Tau b was also visible in the data, but only in discrete emission lines and not in continuum. On 2013 January 2 UT we obtained deeper SNIFS observations of the FW Tau system to increase the $\mathrm{S} / \mathrm{N}$ of our detection. We acquired a total of $1 \mathrm{hr}$ of data (two 30 minute cubes) at an airmass of 1.01. The seeing was excellent, with CFHT's DIMM reporting values of 0.'4-0'.6. We once again recovered the companion in emission; Figure 3 shows SNIFS images of the system on and off the $\mathrm{H} \alpha$ line. The PSF from FW Tau AB contaminates our spectrum of the companion, so for both data sets (2011 October and 2013 January) we extracted the spectrum of FW Tau b using aperture photometry and then fit and subtracted our spectrum of the primary to remove its contaminating flux. (This implicitly assumes the spectra of FW Tau $\mathrm{AB}$ and FW Tau $\mathrm{b}$ differ.) The residuals reveal a rich spectrum of emission lines in FW Tau b, most of which are not seen in the spectrum of FW Tau AB, but our data do not detect the continuum.

Our spectrum of FW Tau AB was flux-calibrated using standards from each night; we estimate the uncertainty to be $\sim 10 \%$ based on observations of the same star targeted on six nights as part of a separate program with SNIFS (Mann et al. 2013; Lépine et al. 2013). FW Tau AB is a variable star, so this approach is more accurate than using archival photometry for flux calibration. The spectra of FW Tau b were then absolutely flux calibrated using the ratio of $\mathrm{H} \alpha$ line strengths between FW 

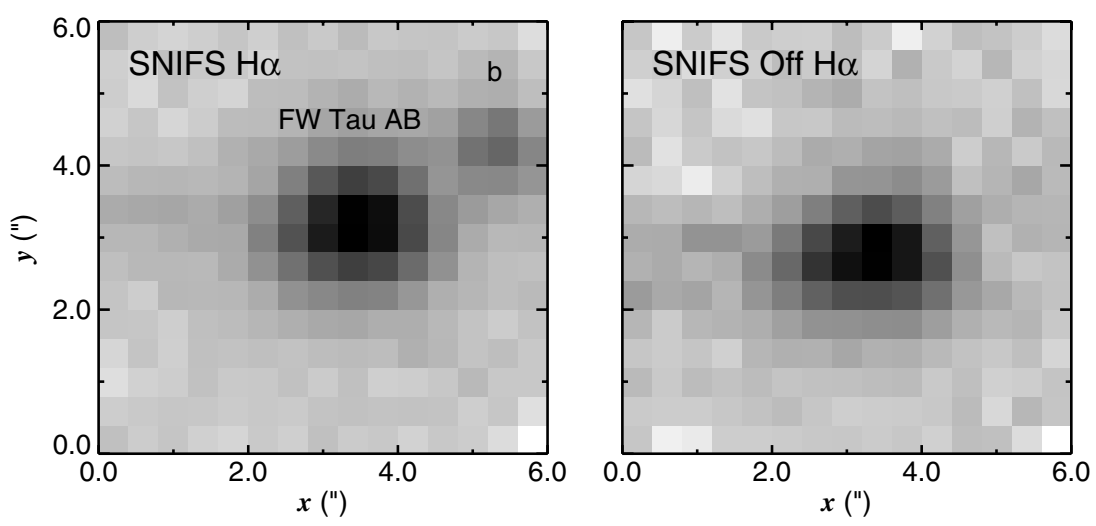

Figure 3. Seeing-limited SNIFS IFU images from 2013 of FW Tau AB+b on and off the $\mathrm{H} \alpha$ line. FW Tau b is detected in emission (left) but is not seen in the adjacent continuum (right). The PSF is slightly elongated in the horizontal direction, probably from telescope windshake.

Tau AB and b (20.7 for our 2011 October data, 15.9 for our 2013 January data).

\subsection{Keck/NIRC2 Imaging of FW Tau ABb}

On 2013 February 4 UT we imaged FW Tau ABb with Keck II/NIRC2 using NGSAO to check for long-term variability in the system. Seeing was highly variable throughout the night, ranging from $\sim 0^{\prime} \cdot 6-2^{\prime \prime}$. We obtained five images in the narrow camera mode $\left(10^{\prime \prime} .2 \times 10^{\prime \prime} 2\right.$ field of view) with integration times of $8.5 \mathrm{~s}$ per frame with the $K^{\prime}$ filter. In all of the images FW Tau AB is easily resolved and FW Tau b is visible. Each image was dark-subtracted and flat-fielded after removing bad pixels and cosmic rays. Optical distortions were corrected using the narrow camera distortion solution created by B. Cameron (2007, private communication).

Photometry and astrometry for FW Tau AB is calculated by fitting an analytic model composed of three elliptical Gaussians to each component as described in Liu et al. (2008). We measure a flux ratio of $\Delta K^{\prime}=0.091 \pm 0.011 \mathrm{mag}$, a separation of $97.3 \pm 2$ mas, and P.A. of $316.7 \pm 0.4$, which represent the mean and standard deviation from the five images. We adopt the sky orientation on the detector and pixel scale from Yelda et al. (2010). Our data show significant outward orbital motion compared to the Hubble Space Telescope (HST) astrometry of $75 \pm 5$ mas and $3.4 \pm 3^{\circ}$ from White \& Ghez (2001).

Relative photometry between FW Tau AB and b is computed in a similar fashion. A model of three elliptical Gaussians is fit to the tertiary, yielding a relative flux ratio of $\Delta K^{\prime}=6.3 \pm$ 0.4 mag between the A-B pair and the outer companion. Despite these large uncertainties caused by poor seeing conditions, our photometry is consistent with that from Kraus et al. (2014) at the $1 \sigma$ level, indicating no substantial $K$-band variability between 2008 and 2013. We measure a separation of $2307 \pm 4$ mas and P.A. of $296^{\circ} 0 \pm 0.10$ relative to FW Tau A, in agreement with the astrometry from White \& Ghez (2001, with an updated P.A. from Kraus et al. 2014).

\section{RESULTS}

\subsection{Spectral Types and Reddenings for the Primary Stars}

Accurate reddening measurements are needed to correct for possible extinction in the spectra of companions. We fit our (unresolved) SNIFS spectra of ROXs 42B AB and FW Tau $\mathrm{AB}$ for spectral type and visual extinction $\left(A_{V}\right)$ using optical templates from Pickles (1998) for K3-K7 types and mean-combined Sloan Digital Sky Survey templates from
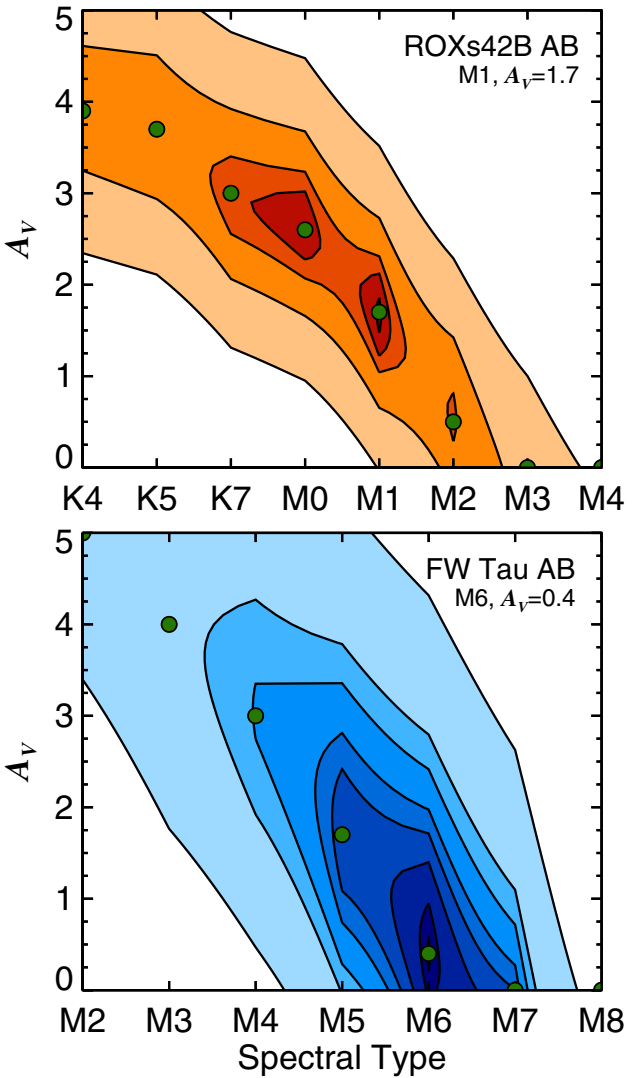

Figure 4. $\chi^{2}$ contour plots showing the relative goodness-of-fit of spectral type and reddening for our SNIFS spectra of ROXs 42B AB (top) and FW Tau AB (bottom). Template spectra are from Pickles (1998) for K dwarfs and Bochanski et al. (2007) for M dwarfs (see Section 3.1). Green circles indicate the best-fit reddening at each spectral type (displayed in Figure 5). The best-matches to the $6000-8500 \AA$ region are $\left\{\mathrm{M} 1 \pm 1, A_{V}=1.7_{-1.2}^{+0.9} \mathrm{mag}\right\}$ for ROXs $42 \mathrm{~B} \mathrm{AB}$ and $\left\{\mathrm{M} 6 \pm 1, A_{V}=0.4_{-0.4}^{+1.3} \mathrm{mag}\right\}$ for $\mathrm{FW}$ Tau $\mathrm{AB}$. Note the strong covariance between reddening and optical spectral type.

(A color version of this figure is available in the online journal.)

Bochanski et al. (2007) for M0-M9 types. The reddening curve of Fitzpatrick (1999) was used with $A_{V} / E(B-V)=3.1$. The spectra were fit using $\chi^{2}$ minimization between $6000-8500 \AA$ by reddening each template in steps of $0.1 \mathrm{mag}$ in $A_{V}$, interpolating onto the wavelength grid of our science spectrum, and scaling to the observed spectrum using the maximum likelihood method described in Bowler et al. (2009). The results are shown in Figures 4 and 5; as expected, there is a strong covariance 

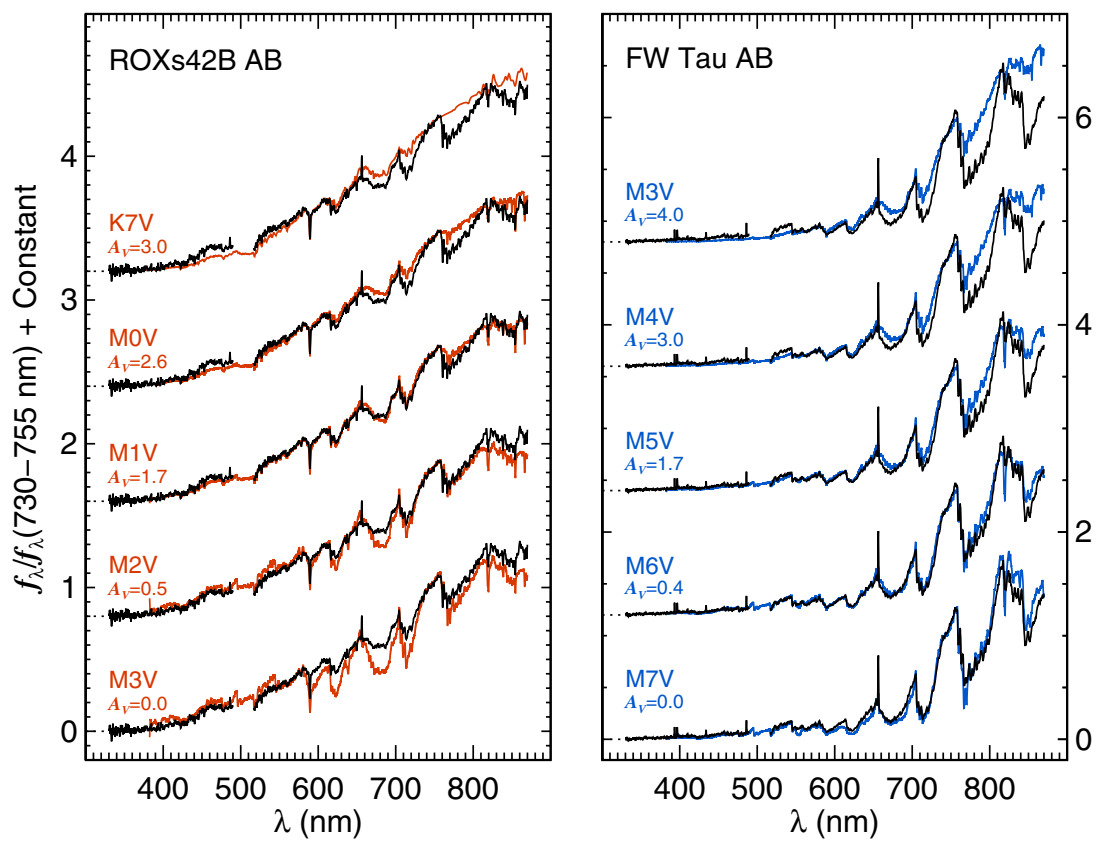

Figure 5. Comparison of the best-fitting reddened optical templates to our SNIFS spectra ROXs 42B AB and FW Tau AB for each spectral type (see Figure 4). The best match is a moderately reddened M1 template for ROXs 42B AB and a slightly reddened M6 template for FW Tau AB. The spectra are normalized between 7300 and $7550 \AA$ and offset by a constant.

(A color version of this figure is available in the online journal.)

between spectral type and extinction, with earlier types being more strongly reddened than later types to match the same slope. The best-fitting spectrum to ROXs $42 \mathrm{~B}$ AB is M1 \pm 1 with a modest reddening of $A_{V}=1.7_{-1.2}^{+0.9}$ mag. Here we adopt an uncertainty of one subclass for the spectral type and the corresponding $A_{V}$ range for the extinction. (Our errors are estimated ranges rather than $1 \sigma$ confidence intervals.) Our bestfit spectral type is comparable to M0 \pm 1 from Bouvier \& Appenzeller (1992). For FW Tau AB, we find a spectral type of M6 \pm 1 and $A_{V}=0.4_{-0.4}^{+1.3} \mathrm{mag}$; this compares well with the measurement of M5.5 \pm 0.5 from Briceño et al. (1998) and the reddening of $A_{V}=0.00-0.83 \mathrm{mag}$ derived by White \& Ghez (2001) for the system based on photometry. FW Tau AB exhibits Balmer emission lines ( $\mathrm{H} \alpha$ through $\mathrm{H} 10$ ) and strong $\mathrm{Ca} H+K$ emission, both signs of strong chromospheric activity associated with youth.

We use the same fitting scheme to revisit the spectral types and reddening values of the primary stars GSC 6214-210 A and 1RXS J1609-2105 A, whose PMCs we use as comparative templates for ROXs 42B b (Section 3.2.3). Our SNIFS spectrum of GSC 6214-210 A was first published in Bowler et al. (2011) while our spectrum of 1RXS J1609-2105 A is presented here for the first time. The results of the fits are shown in Figures 6 and 7 . The best match to GSC 6214-210 A between 6000-8500 $\AA$ is a K5 \pm 1 template reddened by $A_{V}=0.5_{-0.5}^{+0.2} \mathrm{mag}$. In Bowler et al. (2011), we found a spectral type of K7 \pm 1 but did not account for possible extinction; our revised classification and slight reddening is similar to that found by Bailey et al. (2013). 1RXS J1609-2105 A is an excellent match to the M0 template, and we adopt a spectral type of $\mathrm{M} 0 \pm 1$ with a slight reddening of $A_{V}=0.1_{-0.1}^{+0.3} \mathrm{mag}$ for the system. This agrees with the $\mathrm{M} 0 \pm$ 1 type found by Preibisch et al. (1998) and is slightly later than the $\mathrm{K} 7 \pm 1$ classification made by Lafrenière et al. (2008) based on high-resolution optical spectroscopy.
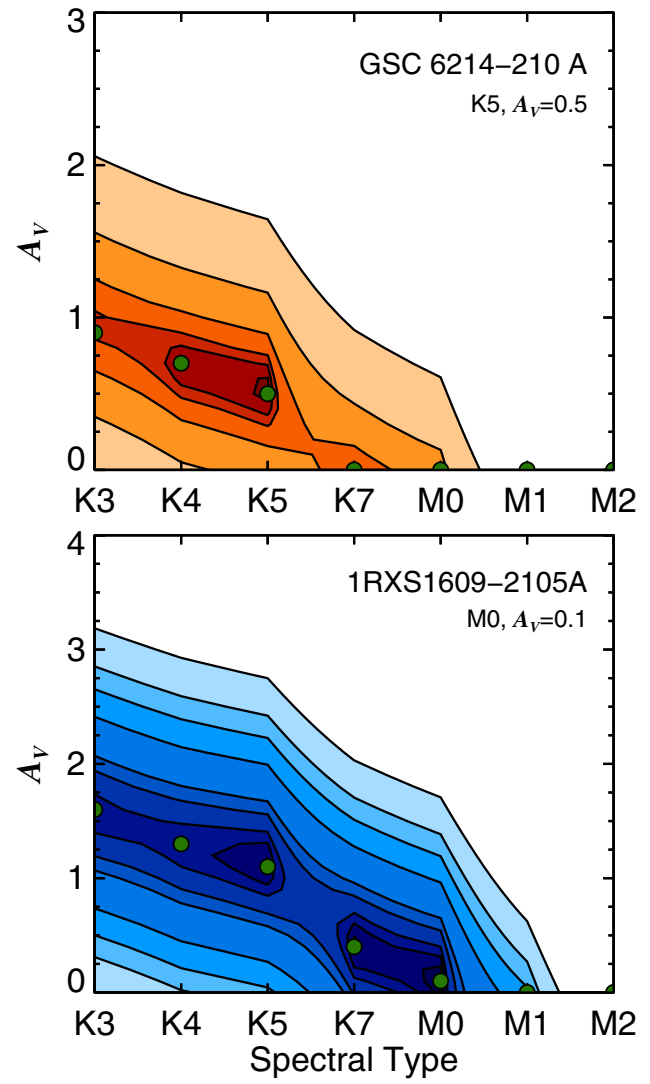

Figure 6. $\chi^{2}$ contour plots for our reddening and spectral type fits to GSC 6214-210 A (top) and 1RXS J1609-2105 A (bottom). See Figure 5 for details. The best fits are $\left\{\mathrm{K} 5 \pm 1, A_{V}=0.5_{-0.5}^{+0.2} \mathrm{mag}\right\}$ for GSC $6214-210 \mathrm{~A}$ and $\left\{\mathrm{M} 0 \pm 1, A_{V}=0.1_{-0.1}^{+0.3} \mathrm{mag}\right\}$.

(A color version of this figure is available in the online journal.) 

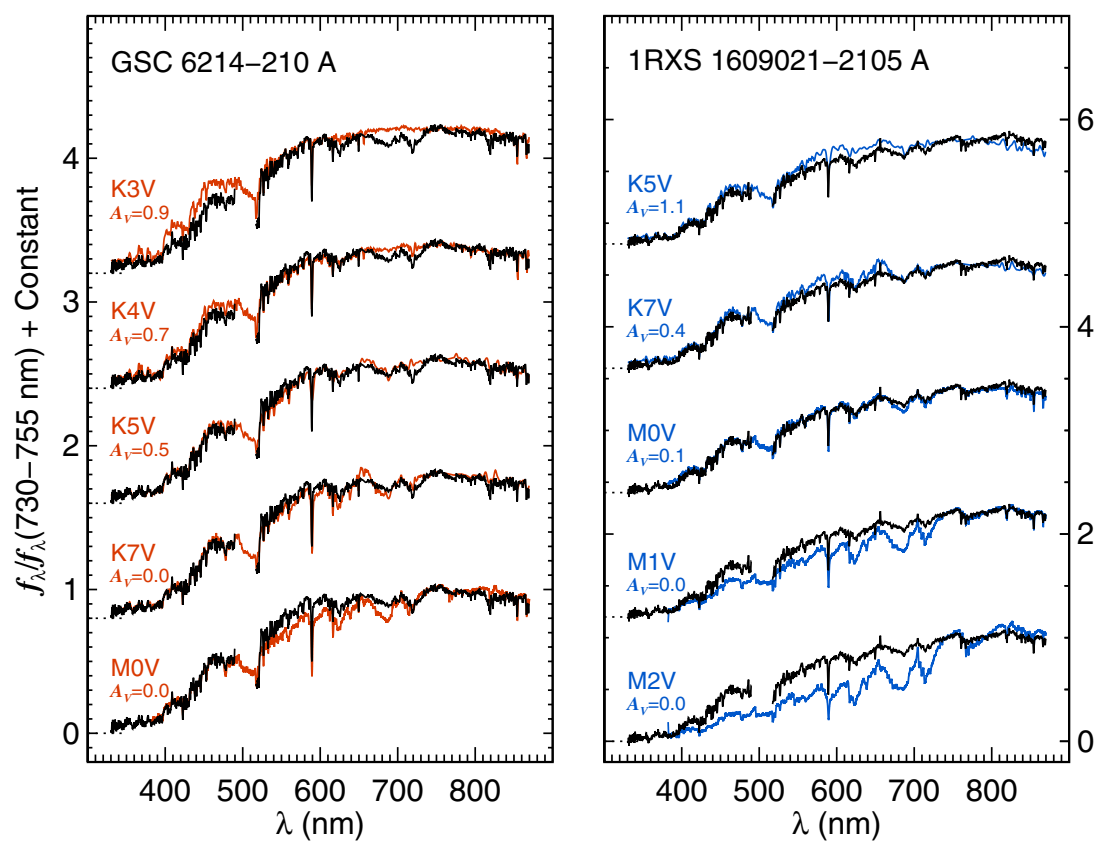

Figure 7. SNIFS spectra of GSC 6214-210 A and 1RXS J1609-2105 A compared to the best-fit reddened optical templates (see Figure 6). A slightly reddened K5 spectrum is somewhat preferred over the unreddened K7 type we found for GSC 6214-210 A in Bowler et al. (2011). The best match to 1RXS J1609-2105 A is the M0 template. The spectra are normalized between 7300 and $7550 \AA$ and offset by a constant.

(A color version of this figure is available in the online journal.)

\subsection{PMC Near-IR Spectral Types}

Our new NIR spectra of GSC 6214-210 B, SR 12 C, ROXs $42 \mathrm{~B} \mathrm{~b}$, and FW Tau $\mathrm{b}$ are presented in Figure 8. Each bandpass for GSC 6214-210 B, ROXs 42B b, and FW Tau b was flux calibrated to the photometry listed in Kraus et al. (2014), and the spectra were Gaussian smoothed from their native resolution to $R \approx 1000$. Our spectrum of SR $12 \mathrm{C}$ was obtained with IRTF/SpeX, which simultaneously samples the 1-2.5 $\mu \mathrm{m}$ region and produces reliable spectral slopes consistent with photometry (Rayner et al. 2009), so no relative flux calibration was performed for that object. We smoothed the spectrum of SR $12 \mathrm{C}$ to $R \approx 500$ to increase the signal-to-noise, although it is still rather modest in $J$-band $(\sim 30)$.

The de-reddened spectra are overplotted in gray in Figure 8, which assumes the same reddening that we found for the primary stars in Section 3.1. Lacking an optical spectrum for SR $12 \mathrm{AB}$, we use extinction values from the literature for that system. A large range of estimates exist: Sartori et al. (2003) cite $0.0 \mathrm{mag}$ based on the $V-I_{C}$ color index of SR $12 \mathrm{AB}$; Marsh et al. (2010) derive a value of $2.3 \pm 1.6 \mathrm{mag}$ from fitting reddened stellar models to $1-4 \mu \mathrm{m}$ photometry; and Wahhaj et al. (2010) infer a value of $7.1 \mathrm{mag}$ based on the $J-K$ color excess. Kuzuhara et al. (2011) find that de-reddening their spectrum of SR $12 \mathrm{C}$ by $A_{J}=$ $0.5 \pm 0.2$ mag produces a good fit to an M9 template spectrum. Using the empirical relation between $A_{V}$ and $A_{J}$ from Rieke \& Lebofsky (1985), this translates into an optical extinction of $A_{V}=1.7 \mathrm{mag}$. This is similar to the value of Marsh et al. (2010), who used a broad wavelength range for their estimation of SR $12 \mathrm{AB}$, so we adopt $A_{V}=1.7 \mathrm{mag}$ for the SR $12 \mathrm{ABC}$ system in this work.

Except for FW Tau b, which we discuss in Section 3.3, our spectra resemble previously known low-gravity late-M/earlyL type objects with shallow $J$-band alkali absorption features and angular $\mathrm{H}$-band shapes (Lucas et al. 2001; Gorlova et al. 2003; McGovern et al. 2004; Allers et al. 2007). Below we describe each spectrum individually and compare them to the new spectral classification system for young late-M and L dwarfs from Allers \& Liu (2013).

\subsubsection{GSC 6214-210 B: M9.5 \pm 1.0}

Allers \& Liu (2013) use a range of spectral indices to link NIR spectral types to optical classification schemes (e.g., Cruz et al. 2009) for young late-M and L dwarfs. They found that comparing the NIR spectra of young objects to standards in the field leads to NIR types that are later than their optical counterparts by $\sim 1$ subtype, a phenomenon also previously noted for late-M dwarfs (e.g., Luhman et al. 2004; Herczeg et al. 2009). In Bowler et al. (2011), we compared the $J$ - and $H$-band spectra of GSC 6214-210 B to M- and L-type templates in the field from Cushing et al. (2005) and Rayner et al. (2009), and the Upper Scorpius star-forming region from Lodieu et al. (2008). However, the Lodieu et al. study also made use of field templates for part of their classifications, possibly biasing our original classification. Moreover, follow-up spectroscopy of these Upper Sco brown dwarfs has revealed that many appear to be up to $\sim 2$ subclasses earlier at optical wavelengths than the NIR estimates (Herczeg et al. 2009; Biller et al. 2011; Lodieu et al. 2011; Bonnefoy et al. 2013). Here we reassess the L0 \pm 1 NIR spectral type we found for GSC 6214-210 B using the new Allers \& Liu classification sequence, our updated reddening measurement for the primary star, and our new $K$-band spectrum.

The leftmost panel of Figure 9 compares the flux-calibrated and de-reddened 1.2-2.4 $\mu \mathrm{m}$ spectrum of GSC 6214-210 B to the "very low-gravity" spectral sequence of Allers \& Liu (2013). Normalized to the $1.66-1.68 \mu \mathrm{m}$ region, the spectral energy distribution (SED) of GSC 6214-210 B is somewhat redder than the young M8 template but bluer than the L0 template. The M9 spectrum is a reasonable match at $J$ and $H$, but the $K$ band of GSC $6214-210 \mathrm{~B}$ is redder than the template. The right three panels of Figure 9 show similar normalized comparisons for 


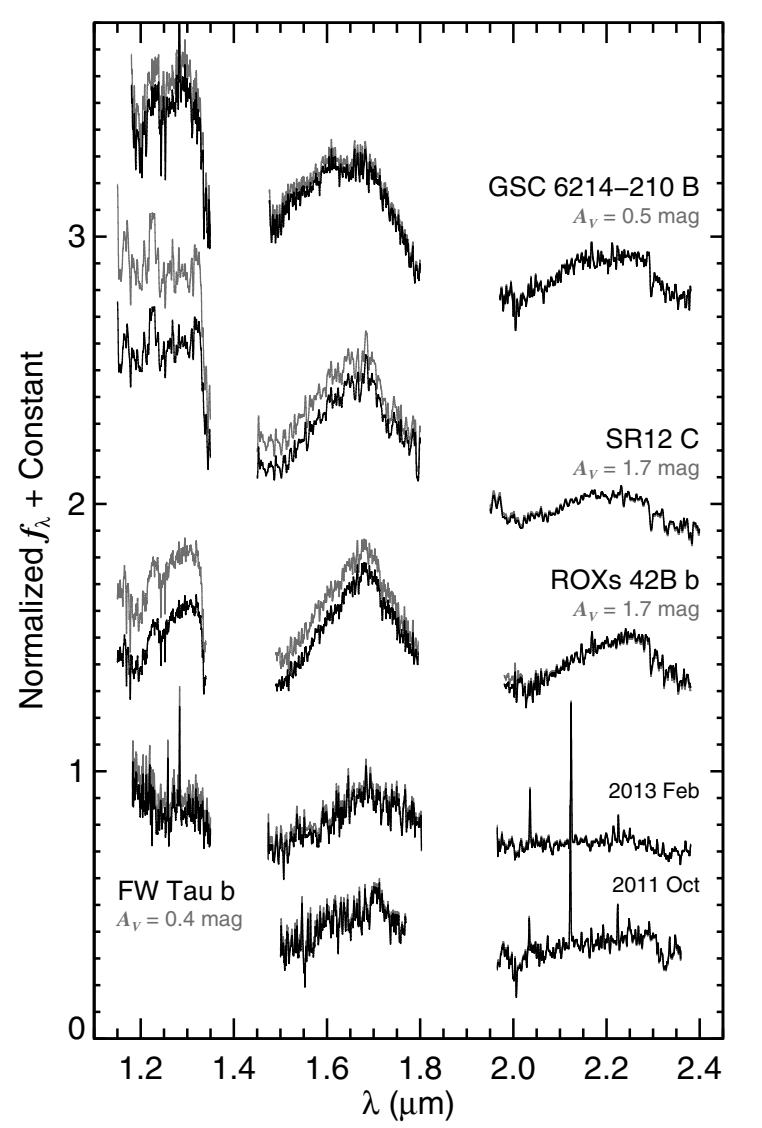

Figure 8. Our near-infrared spectra of very young (2-10 Myr) low-mass (5-15 $M_{\text {Jup }}$ ) companions. The $J$ - and $H$-bands of GSC $6214-210 \mathrm{~B}$ are from Bowler et al. (2011), but the rest are new. The spectra are flux-calibrated to photometry from Ireland et al. (2011), Kuzuhara et al. (2011), and Kraus et al. (2014), and are normalized to the 1.65-1.67 $\mu \mathrm{m}$ region. Overplotted in gray are de-reddened spectra based on extinction values to the primary stars (normalized in $K$ band). GSC 6214-210 B does not show Br $\gamma$ emission, despite its very strong $\mathrm{Pa} \beta$ emission. The angular $H$-bands and weak $J$-band alkali absorption features in these objects are hallmarks of low gravity. Our two epochs of NIR spectra for FW Tau b show a number of strong emission lines from $\mathrm{H}_{2}, \mathrm{~Pa} \beta$, and [Fe II] (see Figure 14), but this object is otherwise relatively flat and featureless. This may be a result of infrared veiling from a warm inner disk or possibly an edge-on disk (see Section 3.3).

the individual bands. GSC 6214-210 B is a good fit to the M9 template in $J$ band, the M8 template in $H$-band, and L1-L2 for $K$ band. The results are summarized in Table 3. Altogether we average the $J H K$ results and adopt a NIR spectral type of M9.5 \pm 1.0 . Interestingly, our $H$-band spectrum of GSC 6214-210 B is significantly broader than the other low-gravity templates in Figure 9. This may point to a somewhat higher surface gravity and hence older age for the system, though the relative change in $H$-band continuum shape does not uniquely track with changes in age (Allers \& Liu 2013). We also note that, despite the strong $\mathrm{Pa} \beta$ emission detected in $J$ band in $2010 \mathrm{July}$, we do not observe similar accretion-induced emission from $\mathrm{Br} \gamma$ in $K$ band in 2012 June. Natta et al. (2004) found that only $2 / 8$ young brown dwarfs showing $\operatorname{Pa} \beta$ emission also showed $\mathrm{Br} \gamma$ emission, so this is not unusual for substellar objects.

\subsection{2. $S R 12 C: M 9.0 \pm 0.5$}

Our spectrum of SR 12 C (Figure 8) exhibits an angular $H$-band shape, an indicator of very low gravity (youth) for lowtemperature objects. The $J$-band region is relatively featureless; we do not detect the $\mathrm{K}_{\mathrm{I}}$ alkali lines at 1.243 and $1.252 \mu \mathrm{m}$,
Table 3

PMC Near-infrared Spectral Types

\begin{tabular}{lccccr}
\hline \hline Object & $1.1-2.4 \mu \mathrm{m}$ & $J$-Band & $H$-Band & $K$-Band & Adopted \\
\hline GSC 6214-210 B & M9 & M9 & M8 & L1-L2 & M9.5 \pm 1 \\
SR12 C & M9 & M8 & M9 & M9-L0 & M9 \pm 1 \\
ROXs 42B b & L1-L2 & M9 & L0-L2 & L1-L2 & L1 \pm 1 \\
\hline
\end{tabular}

which may be due to the modest $\mathrm{S} / \mathrm{N}$ in that region. Strong steam absorption is evident at $1.35 \mu \mathrm{m}$. Figure 10 compares the SED and individual bands of SR 12 C to the Allers \& Liu (2013) sequence. Our de-reddened spectrum of SR $12 \mathrm{C}$ is an excellent match to the M9 spectrum from 1.1-2.4 $\mu \mathrm{m}$. The best matches to the $J, H$, and $K$ bands are the M8, M9, and M9-L0 templates, respectively (Table 3 ). We adopt a NIR classification of M9.0 \pm 0.5 , which agrees with the analysis of Kuzuhara et al. (2011) for their low-resolution NIR spectrum.

$$
\text { 3.2.3. } R O X s 42 B \text { b: } L 1.0 \pm 1.0
$$

The most prominent feature in our spectrum of ROXs $42 \mathrm{~B} \mathrm{~b}$ is the strong angular morphology of the $H$-band, which is especially steep on the blue side compared to other low-gravity substellar objects like SR $12 \mathrm{C}$. The $J$-band region reveals strong $\mathrm{FeH}$ absorption at $1.19-1.20 \mu \mathrm{m}$ and relatively shallow $\mathrm{K}_{\mathrm{I}}$ lines at 1.243 and $1.252 \mu \mathrm{m}$. Compared to the Allers \& Liu sequence (Figure 11), the SED of ROXs 42B b resembles L1-L2 templates. Individual band comparisons are best matched with M9-L2 templates (see Table 3). While the red end of the $H$ band matches M8-L2 objects equally well, the blue end best matches the L1 and L2 templates. Likewise, the depth of the steam absorption near $2 \mu \mathrm{m}$ is similar to the L1 and L2 standards. The $J$ band is bluer than the L2 template but slightly redder than the M9 standard. We emphasize that our comparison in $J$ band suffers the most uncertainty since the reddening of the system, $A_{V}=1.7_{-1.2}^{+0.9} \mathrm{mag}$, is only modestly constrained from fitting the optical spectrum of the primary. While this uncertainty has little impact on the $H$ and $K$ bands, it has a noticeable effect on the $J$-band classification. For $A_{V}=2.6 \mathrm{mag}$, the best-fitting $J$-band type is M9, whereas for $A_{V}=0.5 \mathrm{mag}$, the best match is L0-L1. Altogether, we assign ROXs 42B b a near-infrared spectral type of L1.0 \pm 1.0 .

Figure 12 provides a detailed comparison of ROXs $42 \mathrm{~B} \mathrm{~b}$ with late-type substellar members of other young associations. Lodieu et al. (2008) identified USco J160603.75-221930.0 (hereinafter USco 1606-2219) as a low-mass brown dwarf member of the Upper Scorpius star-forming region and inferred a spectral type of L2 from moderate-resolution near-IR spectroscopy. Optical spectroscopy by Herczeg et al. (2009) revealed an earlier optical type of M8.75 along with evidence of accretion from Balmer continuum excess and extraordinarily strong $\mathrm{H} \alpha$ emission $(\sim 750 \pm 80 \AA)$. ROXs $42 \mathrm{~B}$ b appears to be slightly later than USco 1606-2219 compared to the near-IR spectrum from Lodieu et al., especially in $H$ and $K$ bands, assuming an extinction of $A_{V}=0.0 \mathrm{mag}$ for USco 1606-2219 (Herczeg et al. 2009).

ROXs $42 \mathrm{~B}$ b is a good match in $H$ and $K$ to our prism spectrum of the young Taurus L0 object 2M0437+2331 (Figures 12 and 13). The $J$-band spectrum of ROXs42B b is significantly bluer than $2 \mathrm{M} 0437+2331$ assuming $A_{V}=1.7 \mathrm{mag}$. On the other hand, the spectra are in better agreement if we use the lower bound of the extinction to the system $\left(A_{V}=0.5 \mathrm{mag}\right)$. Here we have assumed zero reddening to $2 \mathrm{M} 0437+2331$, as found by Luhman et al. (2009). Although Alves de Oliveira et al. (2013) 

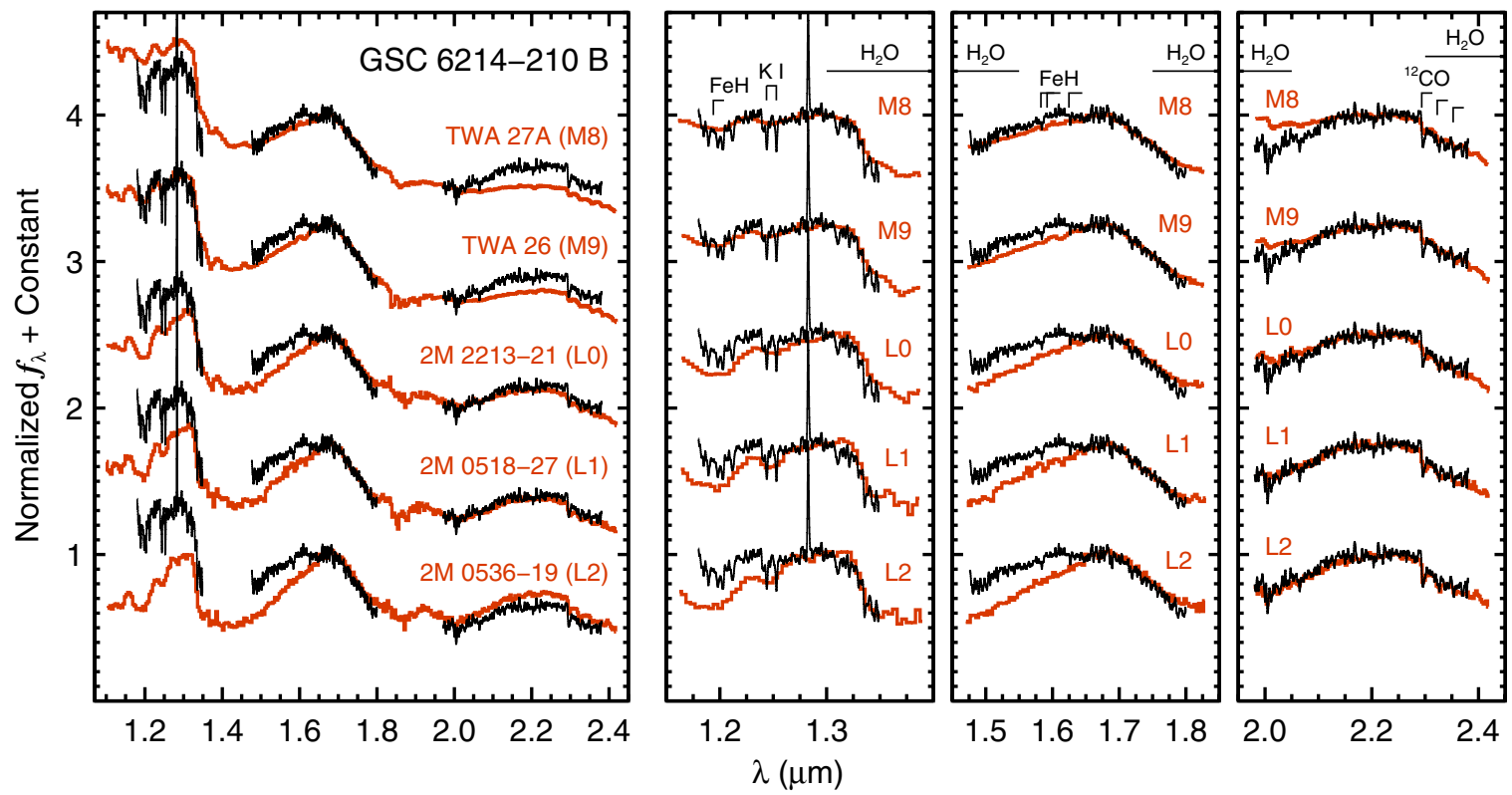

Figure 9. NIR spectral classification of GSC 6214-210 B (black) based on the very low-gravity sequence (red) defined by Allers \& Liu (2013). The left panel shows the flux-calibrated spectrum of GSC 6214-210 B de-reddened by $A_{V}=0.5 \mathrm{mag}$ and smoothed to $R=1000$. The right three panels show the same comparison for the individual $J, H$, and $K$ bands. The SED (which is dominated by the accuracy of the photometry) is somewhat redder than the M9 template. The $J, H$, and $K$ bands match the M9, M8, and L1-L2 templates, respectively; altogether we adopt a NIR spectral type of M9.5 \pm 1.0. The spectra are normalized to the 1.66-1.68 $\mu$ m regions for the first and third panels, and the 1.29-1.31 $\mu \mathrm{m}$ and 2.20-2.25 $\mu \mathrm{m}$ regions for the second and fourth panels, respectively. Major absorption features from $\mathrm{K}$ I, FeH, $\mathrm{H}_{2} \mathrm{O}$, and $\mathrm{CO}$ are labeled.

(A color version of this figure is available in the online journal.)
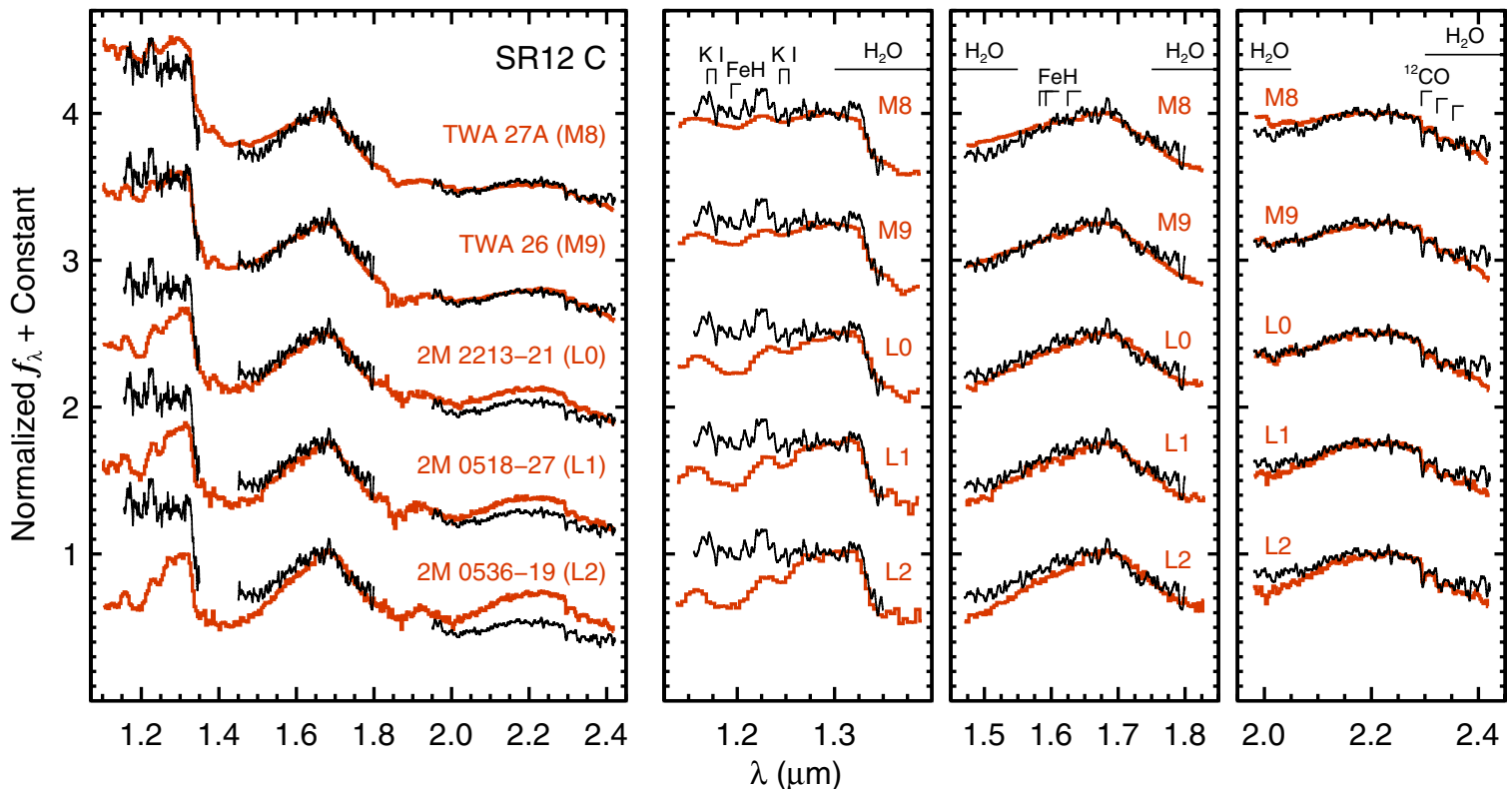

Figure 10. NIR spectral classification of SR 12 C (similar to Figure 9). The M9 template is an excellent fit to our spectrum. The M8, M9, and M9-L0 templates best match the individual bandpasses (from left to right). We adopt a NIR spectral type of M9.0 \pm 0.5 , which is identical to the classification by Kuzuhara et al. (2011) based on a low-resolution spectrum. Our IRTF moderate-resolution spectrum has been smoothed to $R=500$ and de-reddened by $A_{V}=1.7$ mag.

(A color version of this figure is available in the online journal.)

recently suggest a larger reddening value of $A_{V}=2.1-3.3$ mag to 2M0437+2331 based on NIR spectral type matching, we adopt the value from Luhman et al. for this work since it is derived from optical data, which is more sensitive to reddening.

We also compare ROXs 42B b with the young L-type companions AB Pic $b$ (Chauvin et al. 2005; Bonnefoy et al. 2010) and 1RXS J160929.1-210524 b (herinafter 1RXS1609-2105 b; Lafrenière et al. 2008, 2010). Our de-reddened $J$-band spectrum of ROXs $42 \mathrm{~B} \mathrm{~b}$ is slightly bluer than that of the L0 companion AB Pic b (recently reclassified by Allers \& Liu 2013 and Bonnefoy et al. 2013), but the $H$ and $K$ regions appear somewhat redder. Compared with the L4 object 1RXS1609-2105 b, ROXs 42B b is a good match at $H$ and $K$, but 1RXS1609-2105 $\mathrm{b}$ is significantly bluer in $J$. Altogether, our NIR classification of L1.0 \pm 1.0 for ROXs 42B b broadly agrees with the previous classification of these young brown dwarfs. However, the shallow $J$-band slope relative to $2 \mathrm{M} 0437+2331$ and $\mathrm{AB}$ Pic $\mathrm{b}$ may suggest that our reddening estimate of 

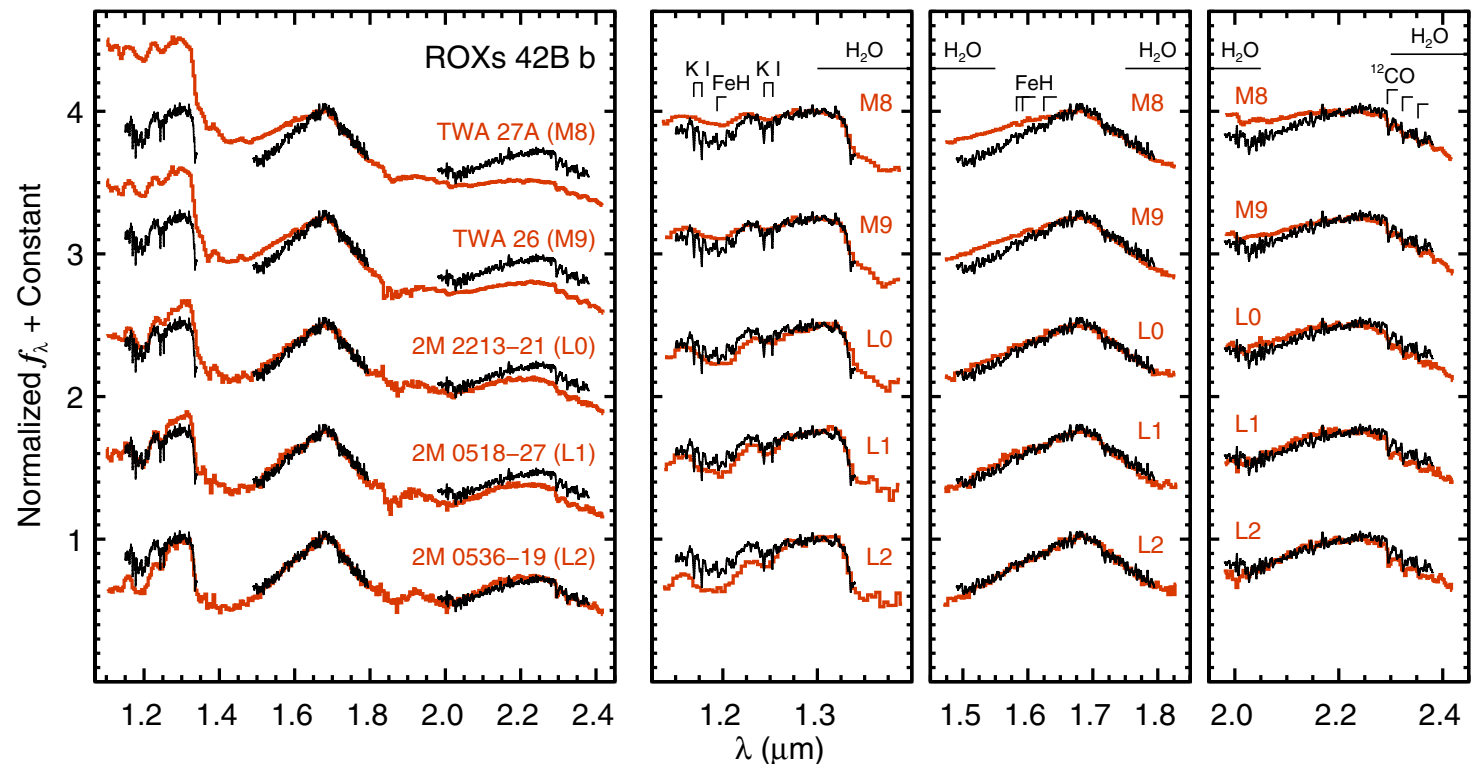

Figure 11. NIR spectral classification of ROXs 42B b (similar to Figure 9). The SED of ROXs42B b is slightly redder than the L1 template (left panel), and the best-matches to the individual bands (right panels) range from M9-L2. Altogether, we adopt a NIR spectral type of L1.0 \pm 1.0 . Our flux-calibrated spectrum has been smoothed to $R=1000$ and de-reddened by $A_{V}=1.7 \mathrm{mag}$.

(A color version of this figure is available in the online journal.)

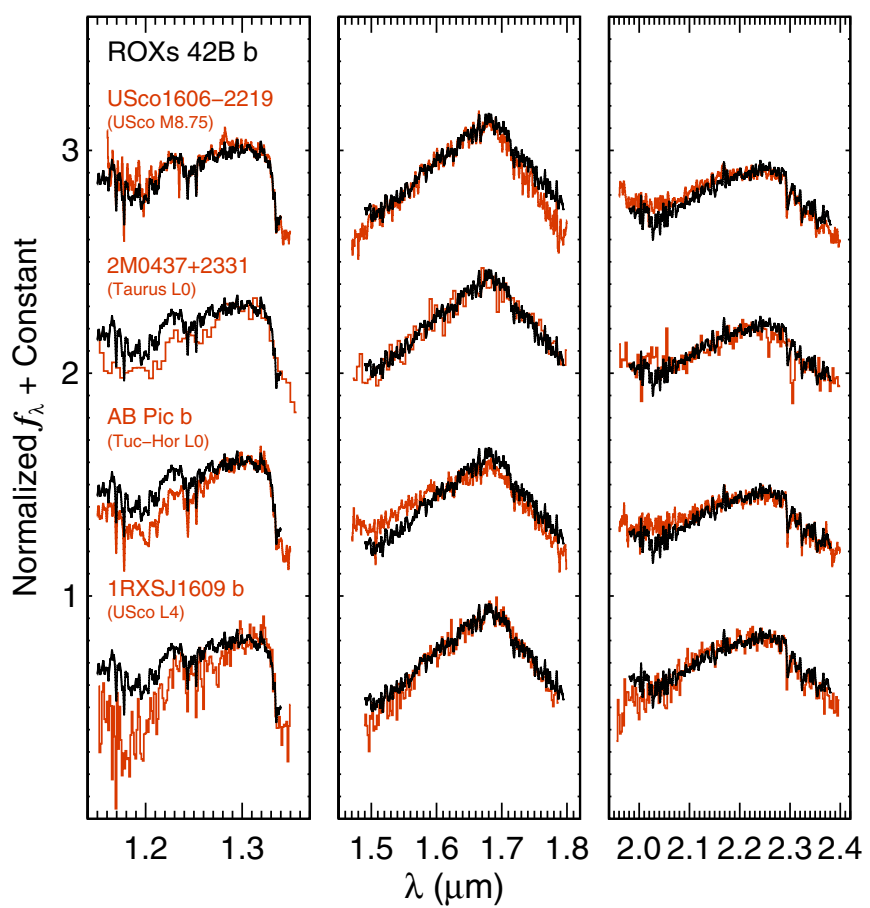

Figure 12. Comparison of our de-reddened spectrum of ROXs $42 \mathrm{~B}$ b to other young substellar objects. Overall, ROXs $42 \mathrm{~B}$ b is most similar to the L0 Taurus member $2 \mathrm{M} 0437+2331$ and the $\sim$ L4 Upper Scorpius companion 1RXS1609-2105 b in $H$ and $K$ bands, and the M8.75 Upper Scorpius brown dwarf USco $1606-2219$ in $J$-band. Note that the $J$-band comparisons are the most prone to uncertainties in our reddening estimate of ROXS 42B AB+b $\left(A_{V}=1.7_{-1.2}^{+0.9} \mathrm{mag}\right)$. The comparison spectra of USco 1606-2219, and AB Pic b are from Lodieu et al. (2008) and Bonnefoy et al. (2010), respectively. The 1RXS1609-2105 b spectrum is from Lafrenière et al. (2008) and Lafrenière et al. (2010). We assume zero reddening to these comparison objects.

(A color version of this figure is available in the online journal.)

$A_{V}=1.7 \mathrm{mag}$ is too large. We also note that Currie et al. (2014) recently presented a $K$-band spectrum of ROXs $42 \mathrm{~B} \mathrm{~b}$ and found a spectral type of M8-L0, which is somewhat earlier than the type we infer from complete $J H K$ spectral coverage.

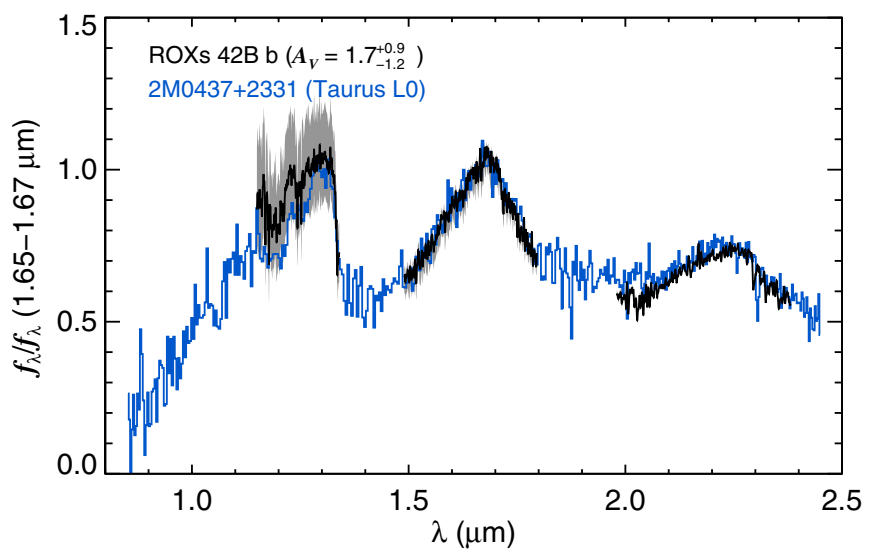

Figure 13. Our de-reddened spectrum of ROXs 42B b (black) bears a close resemblance to the 4-7 $M_{\text {Jup }}$ L0 Taurus member 2M0437+2331 (blue). The gray shaded region shows the range of extinction values for ROXs $42 \mathrm{~B} b$ based on our fits to the primary (Section 3.1). Both spectra are normalized to the $1.65-1.67 \mu \mathrm{m}$ region.

(A color version of this figure is available in the online journal.)

\subsection{Disk Accretion and Outflow Activity from FW Tau b \\ 3.3.1. Overview}

Our spectra of FW Tau b reveal a wealth of permitted and forbidden emission lines spanning the optical through NIR. The optical IFU data from SNIFS are not sensitive enough to detect continuum emission, but prominent emission lines from $4000-9000 \AA$ are visible. The NIR spectrum shows emission from molecular hydrogen and $\mathrm{Pa} \beta$, but the continuum in $J$, $H$, and $K$ bands is relatively flat and featureless. Continuumsubtracted $\mathrm{H}_{2}$ images display slight, but significant, extended emission, indicating spatially resolved structure at small separations $(\lesssim 15 \mathrm{AU})$. These emission lines are commonly seen in Herbig-Haro (HH) flows and their infrared counterparts, molecular hydrogen emission-line objects (MHOs). Together with its extended morphology, this indicates that FW Tau b is a young accreting object driving an outflow. 


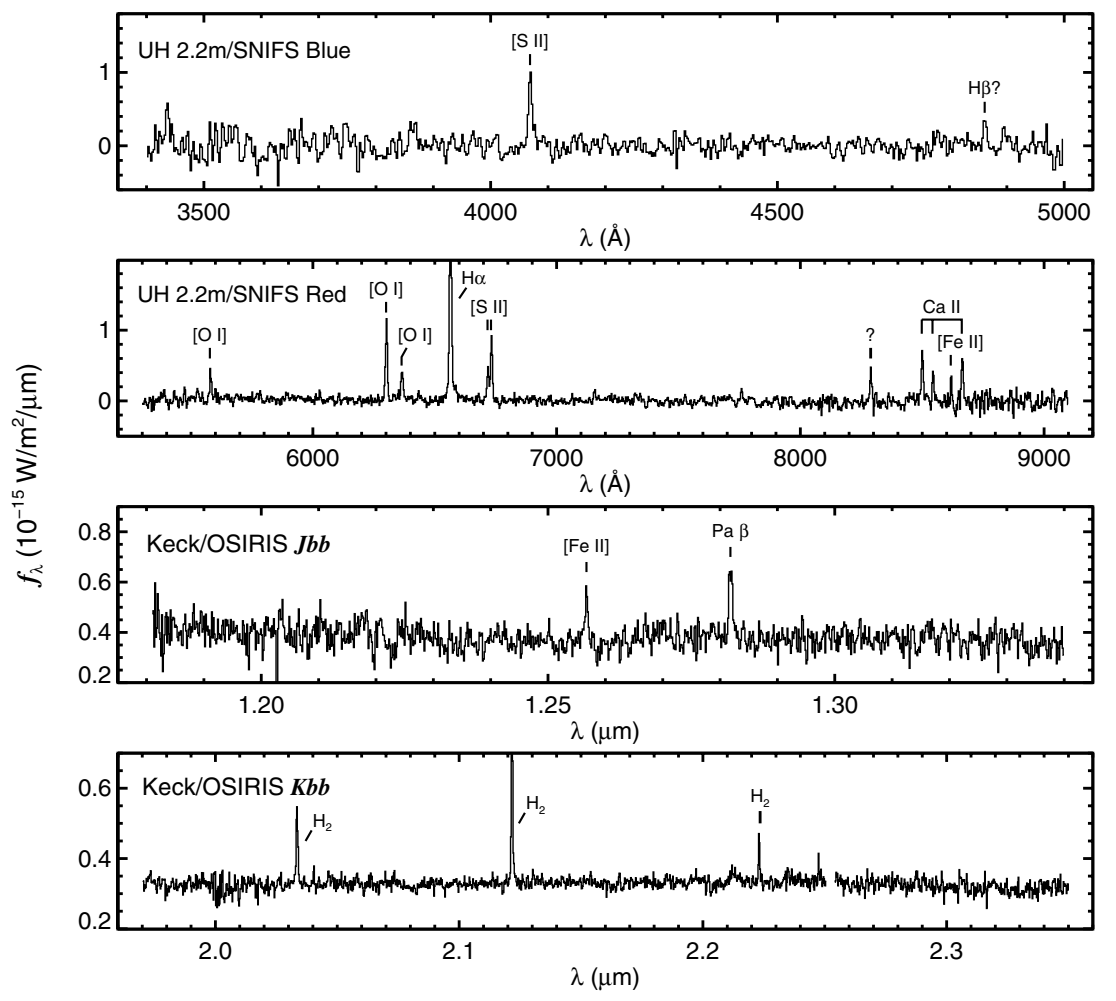

Figure 14. Optical through near-infrared emission line spectrum of FW Tau b. Our SNIFS spectrum (2013 observations; top two panels) shows a wealth of permitted and forbidden emission lines, but does not reach the continuum level. Our $J$ - and $K$-band spectra (2013 observations; bottom two panels) also show emission lines associated with accretion and shock excitation, as well as mostly flat continuum. The spectra are absolutely flux-calibrated and prominent emission lines are labeled. Line fluxes and equivalent widths are listed in Table 4.

\subsubsection{Optical Emission-line Spectrum}

Our flux-calibrated optical spectrum from 2013 January is shown in Figure 14. We detect forbidden emission lines from $\left[\mathrm{S}_{\mathrm{II}}\right] \lambda \lambda 4069,6716,6731 ;$ [O I] $\lambda \lambda 5577,6300,6464 ;$ and [Fe II] $\lambda 8617$, as well as permitted Ca II $\lambda \lambda 8498,8542,8662$, and strong (>290 $\mathrm{A}) \mathrm{H} \alpha$ emission. A possible detection of $\mathrm{H} \beta$ emission is visible, but at weak significance. In addition, we note an emission line at $8286.4 \pm 0.5 \AA$ that is present at both epochs but which we are unable to identify. ${ }^{7}$ We do not reach the continuum level so we provide lower limits on the equivalent widths together with line fluxes in Table 4 . Line strengths are measured by fitting a Gaussian to each emission line using the least-squares curve fitting routine MPFIT (Markwardt 2009), except for the partly blended $\left[\mathrm{S}_{\mathrm{II}}\right] \lambda \lambda 6716,6731$, for which we simultaneously fit two Gaussians. Uncertainties in the line strength are derived in a Monte Carlo fashion by randomly adding noise drawn from a Gaussian distribution with a standard deviation equal to the spectral measurement uncertainty at that wavelength and then refitting the line profile. The mean and associated error from 200 trials are listed in Table 4. To compute lower limits on the equivalent widths, we adopt continuum levels equal to the standard deviation of the data after removing the emission lines $\left(4.8 \times 10^{-16} \mathrm{~W} \mathrm{~m}^{-2} \mu \mathrm{m}^{-1}\right.$ for our 2011 October data, $1.1 \times 10^{-16} \mathrm{~W} \mathrm{~m}^{-2} \mu \mathrm{m}^{-1}$ for our 2013 January data).

Overall, the forbidden emission lines from FW Tau b resemble shock-excited gas typically seen in HH flows (Schwartz 1983;

\footnotetext{
7 Two possibilities from the National Institute of Standards and Technology Atomic Spectra Database (Kramida et al. 2012, http://physics.nist.gov/asd) are Ne II at $\lambda_{\text {air }}=8286.6$ and Fe I at $\lambda_{\text {air }}=8287.3$.
}

Reipurth \& Bally 2001). ${ }^{8}$ Of particular interest is the [Fe II] $\lambda 8617$ line, which is rarer in $\mathrm{HH}$ objects and their sources than [S II] and [O I] lines (Reipurth \& Bally 2001). [Fe II] $\lambda 8617$ emission is generally attributed to collisional excitation from shocks; examples include the HH 34 energy source (Reipurth et al. 1986), the HN Tau (Hartigan et al. 2004) and HH 1 (Nisini et al. 2005) jets, and somewhat more enigmatic young stars such as V1647 Ori (Aspin et al. 2008), MWC 778 (Herbig \& Vacca 2008), and PTF 10nvg (Covey et al. 2011; Hillenbrand et al. 2013).

Raga et al. (1996) find that $\mathrm{HH}$ objects can be divided into low-, intermediate-, and high-excitation classes based on the [O III] $\lambda 5007 / \mathrm{H} \beta$ and $[\mathrm{S} \mathrm{II}] \lambda \lambda(6716+6731) / \mathrm{H} \alpha$ line ratios. While we do not detect the [O III] $\lambda 5007$ line, our [S II] $\lambda \lambda(6716+6731) / \mathrm{H} \alpha$ measurement of 0.36 is comparable to intermediate- to high-excitation HH objects. (Our value is a lower limit, however, since activity and especially accretion from FW Tau b are also likely contributing to the $\mathrm{H} \alpha$ emission). Compared to the sample of $\mathrm{HH}$ objects studied by Raga et al., our measurement of the electron density $\left(\sim 6 \times 10^{3} \mathrm{~cm}^{-3}\right.$; see Section 3.3.7) for FW Tau $b$ is most consistent with the highexcitation sources, which also tend to have large $\left(10^{3}-10^{4} \mathrm{~cm}^{-3}\right)$ values.

The CaII infrared triplet $\lambda \lambda 8498,8542,8662$ has been observed in many young substellar objects (Luhman et al. 2003a;

\footnotetext{
8 We follow the definition of a Herbig-Haro object put forth by Reipurth (1999, p. 4) for his catalog of Herbig-Haro flows: “...flows that extend more than an arcsecond from a star, and which could in principle be studied morphologically using proper coronagraphic techniques, are included in this catalog. Stars with $\mathrm{HH}$ emission lines but no appreciable extent have not been included." Therefore, we do not classify FW Tau b as an HH object since we do not detect extended emission in our (seeing-limited) SNIFS data.
} 
Table 4

FW Tau b Emission Lines

\begin{tabular}{|c|c|c|c|c|c|}
\hline Species & Transition & $\begin{array}{l}\lambda^{\mathrm{a}} \\
(\AA)\end{array}$ & $\begin{array}{l}\text { Date } \\
\text { (UT) }\end{array}$ & $\begin{array}{c}\text { Line Flux } \\
\left(10^{-16} \mathrm{erg} \mathrm{s}^{-1} \mathrm{~cm}^{-2}\right)\end{array}$ & $\begin{array}{c}\mathrm{EW}^{\mathrm{c}} \\
(\AA)\end{array}$ \\
\hline \multirow[t]{2}{*}{ (S II) } & ${ }^{2} P_{3 / 2}^{o}-{ }^{4} S_{3 / 2}^{o}$ & 4068.6 & 2011 Oct 8 & $57 \pm 12$ & $>-101 \pm 17$ \\
\hline & & & 2013 Jan 2 & $8.1 \pm 1.3$ & $>-54 \pm 8$ \\
\hline (OI) & ${ }^{1} S_{0}-{ }^{1} D_{2}$ & 5577.3 & 2013 Jan 2 & $3.9 \pm 1.0$ & $>-18 \pm 6$ \\
\hline \multirow[t]{2}{*}{ (OI) } & ${ }^{1} D_{2}-{ }^{3} P_{2}$ & 6300.3 & 2011 Oct 8 & $11 \pm 2$ & $>-11 \pm 4$ \\
\hline & & & 2013 Jan 2 & $10.4 \pm 1.3$ & $>-75 \pm 8$ \\
\hline (OI) & ${ }^{1} D_{2}-{ }^{3} P_{1}$ & 6363.8 & 2013 Jan 2 & $4.6 \pm 1.0$ & $>-24 \pm 5$ \\
\hline \multirow[t]{2}{*}{$\mathrm{H} \alpha$} & $n=3-2$ & 6562.7 & 2011 Oct 8 & $40 \pm 4$ & $>-67 \pm 5$ \\
\hline & & & 2013 Jan 2 & $35 \pm 2$ & $>-292 \pm 12$ \\
\hline \multirow[t]{2}{*}{ (S II) } & ${ }^{2} D_{5 / 2}^{o}-{ }^{4} S_{3 / 2}^{o}$ & 6716.4 & 2011 Oct 8 & $6 \pm 2$ & $\ldots$ \\
\hline & & & 2013 Jan 2 & $4.4 \pm 0.6$ & $>-24 \pm 4$ \\
\hline \multirow[t]{2}{*}{ (S II) } & ${ }^{2} D_{3 / 2}^{o}-{ }^{4} S_{3 / 2}^{o}$ & 6730.8 & 2011 Oct 8 & $9 \pm 2$ & $>-8 \pm 4$ \\
\hline & & & 2013 Jan 2 & $8.3 \pm 0.6$ & $>-57 \pm 6$ \\
\hline$?$ & & $8286.4 \pm 0.5$ & 2013 Jan 2 & $3.8 \pm 0.7$ & $>-19 \pm 4$ \\
\hline Ca II & ${ }^{2} P_{3 / 2}^{o}-{ }^{2} D_{3 / 2}$ & 8498.0 & 2013 Jan 2 & $6.5 \pm 0.9$ & $>-40 \pm 5$ \\
\hline Ca II & ${ }^{2} P_{3 / 2}^{o}-{ }^{2} D_{5 / 2}$ & 8542.1 & 2013 Jan 2 & $3.5 \pm 0.6$ & $>-18 \pm 4$ \\
\hline (Fe II $)$ & $a^{4} P_{5 / 2}-a^{4} F_{9 / 2}$ & 8617.0 & 2013 Jan 2 & $2.3 \pm 0.5$ & $>-11 \pm 4$ \\
\hline Ca II & ${ }^{2} P_{1 / 2}^{o}-{ }^{2} D_{3 / 2}$ & 8662.1 & 2013 Jan 2 & $5.8 \pm 0.9$ & $>-35 \pm 6$ \\
\hline (Fe II $)$ & $a^{4} D_{7 / 2}-a^{6} D_{9 / 2}$ & 12567 & 2013 Feb 1 & $1.7 \pm 0.4$ & $-4.7 \pm 1.3$ \\
\hline $\mathrm{Pa} \beta$ & $n=5-3$ & 12818 & 2013 Feb 1 & $3.1 \pm 0.4$ & $-8.4 \pm 1.0$ \\
\hline \multirow[t]{2}{*}{$\mathrm{H}_{2}$} & $1-0 S(2)$ & 20338 & 2011 Oct 4 & $0.7 \pm 0.2$ & $-2.0 \pm 0.6$ \\
\hline & & & 2013 Feb 1 & $1.9 \pm 0.2$ & $-5.9 \pm 0.5$ \\
\hline \multirow[t]{2}{*}{$\mathrm{H}_{2}$} & $1-0 S(1)$ & 21218 & 2011 Oct 4 & $4.6 \pm 0.2$ & $-13.6 \pm 0.5$ \\
\hline & & & 2013 Feb 1 & $4.7 \pm 0.2$ & $-14.1 \pm 0.6$ \\
\hline \multirow[t]{2}{*}{$\mathrm{H}_{2}$} & $1-0 S(0)$ & 22233 & 2011 Oct 4 & $1.0 \pm 0.2$ & $-3.1 \pm 0.5$ \\
\hline & & & 2013 Feb 1 & $0.61 \pm 0.14$ & $-1.8 \pm 0.4$ \\
\hline
\end{tabular}

Notes.

a Air wavelengths from the National Institute of Standards and Technology atomic line database (Kramida et al. 2012) are used below $2 \mu \mathrm{m}$; vacuum wavelengths for $H_{2}$ from Tokunaga (2000) are used above $2 \mu \mathrm{m}$.

$\mathrm{b}$ The quoted uncertainties do not take into account the absolute flux calibration error, which we estimate to be $\sim 10 \%$ for our SNIFS data.

c Negative values indicate emission. We do not detect continuum emission in our FW Tau b optical spectra, so our measurements of line fluxes and equivalent widths represent lower limits.

Scholz \& Jayawardhana 2006). Mohanty et al. (2005) argue that for very low-mass objects, the presence of the Ca II $\lambda 8662$ emission line is an strong indicator of accretion. In general, the Ca II infrared triplet traces chromospheric activity in low-mass stars (e.g., Busà et al. 2007), but also appears in accreting stars (e.g., Muzerolle et al. 1998; Fernández \& Comerón 2001) and some HH flows (Reipurth et al. 1986). The relative strengths of the lines can be used to probe the optical depth of the emitting region, which in principle can be used to distinguish between low-densiy outflow or high-density infall material. Optically thin material yields line strengths of 1:9:5, whereas near-equal line strengths imply an optically thick region-a common observation in young accreting stars (e.g., McGregor et al. 1984; Hamann \& Persson 1989). We measure ratios of 1:0.54:0.60, implying an optically thick origin in the form of accretion flows.

Unusually strong $\mathrm{H} \alpha$ emission $(\mathrm{EW}=-454 \pm 224 \AA$ ) was first detected from FW Tau b by White \& Ghez (2001) using narrow-band imaging with $H S T$ in 1997 . They considered, but rejected, the possibility that the emission source was an $\mathrm{HH}$ knot since its appearance was point-like, the neighboring weak-lined TTS FW Tau AB would have been an unlikely origin, and the object was also detected in continuum emission with the $F 814 \mathrm{~W}$ filter. Figure 15 shows the broadband detections and upper limits from White \& Ghez (2001) compared with our SNIFS continuum upper limit. Considering its strong $\mathrm{H} \alpha$ emission at

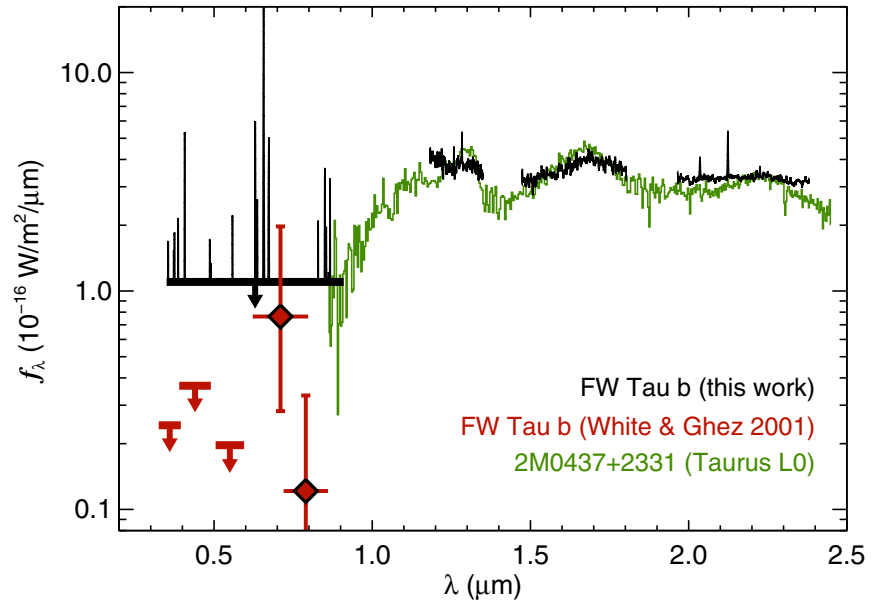

Figure 15. Optical through NIR observations of FW Tau b. Our flux-calibrated 2013 optical spectrum is shown with the $1 \sigma$ continuum upper limit as a thick black horizontal bar at $1.1 \times 10^{-16} \mathrm{~W} \mathrm{~m}^{-2} \mu \mathrm{m}^{-1}$ (the SNIFS data have been smoothed here). Overplotted in red are the White \& Ghez (2001) HST detections $(F 675 W, F 814 W)$ and upper limits $(F 336 W, F 439 W, F 555 W)$ as converted to Johnson-Cousins filter system. Note that the $R$-band detection includes strong $\mathrm{H} \alpha$ emission. For comparison, we also plot our flux-calibrated spectrum of $2 \mathrm{M} 0437+2331$ (no relative scaling is applied to either spectrum). Both $2 \mathrm{M} 0437+2331$ and FW Tau b are members of Taurus so have similar ages and distances. Their apparent NIR magnitudes and colors are similar, but their spectra are somewhat different.

(A color version of this figure is available in the online journal.) 
two epochs spanning $\sim 15 \mathrm{yr}, \mathrm{FW}$ Tau b's high accretion activity appears to be long term and is worthy of continuous monitoring. We note that there is a hint of the neighboring [N II] $\lambda 6583$ line in our data, but, if present, it is too weak and too blended with the $\mathrm{H} \alpha$ line to resolve.

\subsubsection{NIR Continuum Spectrum}

Our OSIRIS spectra of FW Tau b are displayed in Figure 8. As described in Section 2.1, we obtained two epochs of NIR spectroscopy with OSIRIS: $H K$ on 2011 October 4 and $J H K$ on 2013 February 1-3. FW Tau AB is variable in the optical (hence its name), so care must be taken with flux calibration since we do not have simultaneous absolutely calibrated NIR photometry. Indeed, archival sky surveys in the Vizier database indicate $R$-band magnitude variations of $\approx 1$ mag for $\mathrm{FW}$ Tau AB. Photometric variability from stellar flares and star spots is minimal in the near-infrared (e.g., Tofflemire et al. 2011; Davenport et al. 2012; Goulding et al. 2012). Accretion flows, hot spots, extinction variations, and disk heating can result in strong NIR variability (Skrutskie et al. 1996; Carpenter et al. 2001; Eiroa et al. 2002; Rice et al. 2012), but these effects are irrelevant for the Class III primary FW Tau AB (Rebull et al. 2010). The lack of variability of the primary and companion is supported by two epochs of $K$-band relative photometry of FW Tau b by Kraus et al. (2014), which was separated by about two years and showed no changes at the $0.1 \mathrm{mag}$ level. We therefore use the apparent magnitudes of FW Tau b in Kraus et al. to absolutely flux calibrate our spectra.

Overall, the detailed shape of FW Tau b's NIR spectra do not resemble that of a young late-type object as we expected from its photometry and age alone, which is similar to the Taurus L0 object $2 \mathrm{M} 0437+2331$. For example, the $H$-band magnitude and $J-K$ color of $2 \mathrm{M} 0437+2331$ is $16.13 \pm 0.15 \mathrm{mag}$ and $1.94 \pm$ 0.33 mag (2MASS system; Skrutskie et al. 2006), respectively, compared with $16.25 \pm 0.07$ mag and $2.02 \pm 0.10$ for FW Tau b (MKO system; Kraus et al. 2014). The flux calibrated spectra of both objects are shown in Figure 15 for comparison; despite their similar colors, their spectra are quite different. Based on the evolutionary models of Baraffe et al. (2003) and assuming an age of 1-5 Myr, the FW Tau b's expected temperature is 2000-2100 K. For field objects, this corresponds to spectral types of $\sim$ L1-L3 (Golimowski et al. 2004). Instead, the $K$-band spectrum is mostly flat, with a slight decreasing slope beyond $2.3 \mu \mathrm{m}$ in our 2013 data and an apparently stronger feature in our 2011 data between $2.30-2.35 \mu \mathrm{m}$. The $\mathrm{S} / \mathrm{N}$ of the smoothed spectra $(R=1000)$ in Figure 8 are between $80-120$ for our 2013 data and 60-90 for our 2011 data, which would easily reveal CO absorption if present.

The $H$ band spectra are of somewhat lower quality, with smoothed $\mathrm{S} / \mathrm{N}$ values of $\sim 30-50$. They appear triangular in morphology, with the peak at a similar location near $1.7 \mu \mathrm{m}$, but with much shallower continuum slopes than for other young early-L dwarfs. Our $J$-band data have the lowest S/N (25-30 in our smoothed data, $\sim 8$ in our raw data), so the spectral shape should be treated with caution. Nevertheless, the data show a decline beyond $1.3 \mu \mathrm{m}$ as is expected from steam absorption in a cool atmosphere, but the rising flux below $1.25 \mu \mathrm{m}$ is unusual and may be a result of poor $\mathrm{S} / \mathrm{N}$.

\subsubsection{Near-IR Veiling?}

One explanation for the weak photospheric features is that they have been filled in ("veiled") by excess continuum and/ or line emission from accretion activity or a warm inner disk. This phenomenon was first recognized in the optical spectra of accreting $\mathrm{T}$ Tauri stars as an additional warm continuum component superimposed on an otherwise typical stellar spectrum, resulting in weaker-than-expected absorption lines (e.g., Basri \& Bertout 1989; Basri \& Batalha 1990; Hartigan et al. 1990; Batalha \& Basri 1993). In the UV/optical, the excess emission is thought to originate from shocks on the stellar surface caused by magnetospheric accretion (e.g., Calvet \& Gullbring 1998). A similar phenomenon occurs in the infrared as a result of reprocessed light from an inner disk, adding continuum emission that can weaken or completely mask otherwise strong photospheric lines (Luhman \& Rieke 1998, 1999; Folha \& Emerson 1999; Johns-Krull \& Valenti 2001; McClure et al. 2013). In addition to continuum excess, emission features from a warm $(>1000 \mathrm{~K})$ inner disk or stellar winds can also contribute to the diminished infrared absorption lines, the most notable being the first overtone bands of $\mathrm{CO}$ at $2.3 \mu \mathrm{m}$ (Carr et al. 1993; Casali \& Eiroa 1996; Najita et al. 1996; Fernández \& Comerón 2001; Berthoud et al. 2007).

Is veiling from a warm disk or accretion flow plausible for $\mathrm{FW}$ Tau b? Based on thermal photometry of young ( $\lesssim 5 \mathrm{Myr})$ brown dwarfs, Liu et al. (2003) found that the inner regions $\left(\approx 2 R_{*}\right)$ of brown dwarf disks can reach temperatures of $\sim 1200 \mathrm{~K}$, similar to those for $\mathrm{T}$ Tauri stars. This manifests as excess emission beyond $\sim 3 \mu \mathrm{m}$ and, for particularly hot disks, at $K$ band (e.g., Luhman 1999; Muench et al. 2001; Liu et al. 2003). Moreover, brown dwarfs with especially high accretion rates $(\mathrm{d} M / \mathrm{d} t \gtrsim$ $10^{-9} M_{\odot} \mathrm{yr}^{-1}$ ) can have accretion hot spots that dominate over the photospheric flux (Mayne \& Harries 2010). This suggests that despite their cool temperatures, brown dwarfs can still experience veiling similar to their higher-mass counterparts. For FW Tau b, we measure a mass accretion rate of $\log (\dot{M})=$ $-11.0 \pm 1.3 M_{\odot} \mathrm{yr}^{-1}$ (Section 3.3.8); although this is below the extreme accretion rates required to dominate the near-IR SED, it might produce partial veiling apparent in our spectra.

In a study of 110 Class I protostars, Connelley \& Greene (2010) found a correlation between CO emission, high veiling (defined as veiling to the point where no photospheric absorption features are detected), and $\mathrm{Br} \gamma$ emission, which is probably caused by large mass accretion events (e.g., Calvet et al. 1991). For FW Tau b, we do not detect $\mathrm{Br} \gamma$ emission, which is without exception observed by Connelley et al. when $\mathrm{CO}$ is seen in emission. Nevertheless, the $\mathrm{Pa} \beta$ and strong $\mathrm{H} \alpha$ emission indicate that FW Tau $\mathrm{b}$ is actively accreting, supporting the idea that strong veiling could be a source of the weak near-IR features. In general, near-IR veiling is characteristic of Class I objects (e.g., Greene \& Lada 1996) and HH sources (Reipurth \& Aspin 1997; Davis et al. 2011), pointing to a young evolutionary stage for FW Tau b.

\subsubsection{An Edge-on Disk?}

Another interpretation is that FW Tau b is a low-mass star or brown dwarf with an edge-on disk. An earlier spectral type would help explain the lack of low-temperature photospheric features, and a nearly edge-on disk orientation could explain its faint optical and near-IR brightness.

Several edge-on disks around $\mathrm{T}$ Tauri stars and young brown dwarfs are known. Their atypical orientations produce distinct observational signatures compared to more common inclinations. Deep optical and NIR imaging often reveals dark, heavily extincted lanes intersecting an extended disk seen in scattered light (e.g., HV Tau C: Monin \& Bouvier 2000, Stapelfeldt et al. 2003; LkH $\alpha$ 263C: Jayawardhana et al. 2002, 


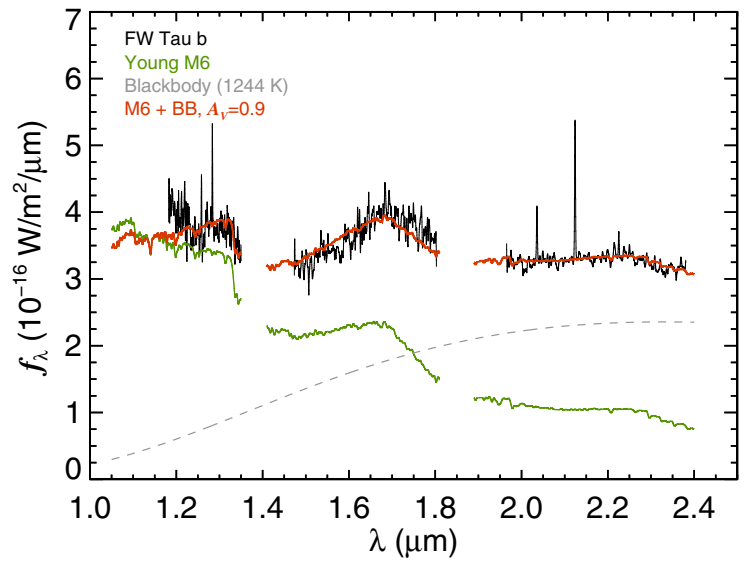

Figure 16. Our NIR spectrum of FW Tau b is reasonably well-fit with a modestly reddened late-M dwarf photosphere (green) and a hot blackbody component (dashed gray). The total emission (red) reproduces the shape of our flux-calibrated spectrum from 1.2 to $2.4 \mu \mathrm{m}$. Among the very low-gravity spectral templates from Allers \& Liu (2013), the young M6 brown dwarf TWA 8B (originally from Allers et al. 2009) provides the best match together with a $1244 \mathrm{~K}$ blackbody and modest reddening of $A_{V}=0.9$ mag. This suggests that the edge-on disk hypothesis is plausible, though more observations will be needed to distinguish these scenarios. Note that emission lines were removed from our spectrum of FW Tau $b$ for this exercise.

(A color version of this figure is available in the online journal.)

Chauvin et al. 2002). Although this morphology is not universal (Huélamo et al. 2010), it is the most telling signature of an edge-on configuration. The SEDs of edge-on disks generally exhibit double-peaked shapes with a broad trough at $\sim 5-20 \mu \mathrm{m}$ (Duchêne et al. 2010) and, at high disk inclinations, the $10 \mu \mathrm{m}$ silicate feature is usually seen in absorption instead of emission (Watson et al. 2004; Luhman et al. 2007).

To test whether an earlier spectral type can plausibly fit our NIR spectrum of FW Tau b, we created a simple model consisting of photospheric and blackbody emission from a single temperature disk. We used the M0-M4 near-infrared spectra from Kirkpatrick et al. (2010) and the "very low-gravity" M5-L3 near-infrared standards from Allers \& Liu (2013) as photosphere templates. Each template was first flux-calibrated to FW Tau b's $J$-band magnitude, which will be minimally affected by warm disk emission. Using the AMOEBA downhill simplex algorithm (Press et al. 2007), we fit a blackbody curve and photosphere template to our $1.1-2.3 \mu \mathrm{m}$ spectrum after applying $A_{V}=0.4$ mag of reddening to match that of FW Tau AB. In addition to the blackbody scale factor and temperature, we allowed a third parameter of additional reddening to the system to vary to account for possible additional extinction from the disk itself. Our spectrum of FW Tau b is well-reproduced with modestly reddened (total $A_{V}=0.80-1.2 \mathrm{mag}$ ) late-M dwarf templates (M5-M8) with a hot $\left(T_{B B}=1200-1330 \mathrm{~K}\right)$ disk. At later spectral types of L0-L3, the fit is somewhat worse $\left(\chi_{v}^{2} \approx\right.$ 5-7 compared to $\approx 2$ for late-M types, where $v=4268$ degrees of freedom), but not implausible. The bet-fit (M6, $T_{B B}=1244 \mathrm{~K}$, $A_{V}=0.9 \mathrm{mag}$ ) is shown in Figure 16. The blackbody scale factor $\left(=R^{2} / d^{2}\right)$ corresponds to an area of $1.6 R_{\odot}^{2}$ for the emitting region assuming a distance of $145 \mathrm{pc}$. Although this simple model neglects other important factors like disk scattering and geometry, it shows that an underluminous late-M-type spectrum together with a warm disk might also explain the properties of this companion.

In this edge-on disk scenario, the earlier spectral types of M5-M8 correspond to effective temperatures of $\sim 2700-3100 \mathrm{~K}$ based on the scale from Luhman et al. (2003b), which assumes young $\mathrm{M}$ dwarfs have temperatures intermediate between field stars and giants. At the system age of $\sim 2 \mathrm{Myr}$, these temperatures correspond to masses of $\sim 30 M_{\text {Jup }}$ to $0.15 M_{\odot}$ based on the evolutionary models of Baraffe et al. (1998).

It is interesting to note that the few known mid- to late$\mathrm{M}$ dwarfs with edge-on disks like TWA 30B (Looper et al. 2010) and 2MASS J04381486+2611399 (Luhman et al. 2007) show deep photospheric absorption features superimposed on otherwise peculiar (and time varying for TWA 30B) SEDs. For example, both of these objects show pronounced $2.3 \mu \mathrm{m} \mathrm{CO}$ absorption, whereas FW Tau b has a flat $K$-band spectrum. This may hint that both an edge-on disk and veiling may be present in FW Tau b.

We can also examine the fraction of flux diminished by edgeon disks in these systems compared to unobscured stars with the same age and spectral type. TWA $30 \mathrm{~B}$ is $\sim 5$ mag fainter in $K$ band than its companion, TWA 30A, despite having an earlier spectral type (M4.0 \pm 0.5 versus M5 \pm 1 ; Looper et al. 2010). This implies at least 5 mag of $K$-band extinction caused by its edge-on disk. Compared to other M7.25 objects in Taurus from Luhman (2004b), 2MASS J04381486+2611399 is fainter in $K$ band by $\sim 1.5-3$ mag. Turning to FW Tau b, our best-fit spectral types in the edge-on disk scenario are M5-M8. This implies $\sim 2.5-5.5 \mathrm{mag}$ of $K$-band extinction compared with Taurus objects from Kraus et al. (2006) and Luhman (2004b). The amount of extinction needed to explain the faintness of FW Tau $\mathrm{b}$ is therefore consistent with other known edge-on disks around $\mathrm{M}$ stars, suggesting a similar disk geometry around FW Tau $\mathrm{b}$ is plausible.

\subsubsection{NIR Emission-line Spectrum}

Several emission lines are visible in the NIR spectrum of FW Tau b (Figure 14). In $K$ band, three low-excitation $(v=1)$ $\mathrm{H}_{2}$ rovibrational lines are present at $2.034 \mu \mathrm{m}, 2.122 \mu \mathrm{m}$, and $2.224 \mu \mathrm{m}$. As described in detail in Beck et al. (2008), several excitation mechanisms can give rise to low- $v \mathrm{H}_{2}$ transitions: shock-excited gas at temperatures of several thousand Kelvin (e.g., Garatti et al. 2006), UV-pumped fluorescence by Ly $\alpha$ emission (Black \& Dishoeck 1987), or stellar heating of an inner disk by high-energy photons (Maloney et al. 1996). The morphology of the $\mathrm{H}_{2}$ emission is spatially extended (Section 3.3.6), so at least some of it appears to be a result of shock-excited outflow activity. UV fluorescence is unlikely because of the absence of emission from higher $\mathrm{H}_{2}$ transitions in our spectra (Giannini et al. 2002, 2004). Unfortunately, we cannot measure reddening and gas temperatures using line ratios since we only detect $v=1$ lines; follow-up spectroscopy with wavelength coverage beyond $2.4 \mu \mathrm{m}$ would enable a more detailed analysis of the excitation conditions if the $\mathrm{H}_{2}$ quadruple emission is present (e.g., Gautier et al. 1976; Nisini et al. 2005).

The $J$-band region shows emission from $\mathrm{Pa} \beta$ and [Fe II] at $1.257 \mu \mathrm{m}$. The [Fe II] emission originates from hot, dense gas at much a higher temperature $\left(\sim 10^{4} \mathrm{~K}\right)$ than the $\mathrm{H}_{2}$ emission (Hollenbach \& McKee 1989; Nisini et al. 2002; Davis et al. 2003; Takami et al. 2006), which dissociates at $\sim 4000 \mathrm{~K}$ (e.g., Lepp \& McCray 1983). Together these lines imply a stratification of gas temperatures and shock speeds at small spatial scales $(\lesssim 15 \mathrm{AU})$. We note that the $1.644 \mu \mathrm{m}[\mathrm{Fe}$ II] line may appear weakly in our 2013 data, but it is not strong enough to unambiguously identify above the pseudocontinuum. If confirmed, the ratio of the $1.257 \mu \mathrm{m}$ and $1.644 \mu \mathrm{m}$ lines can be used to probe extinction to the emitting region (Gredel 1994). 

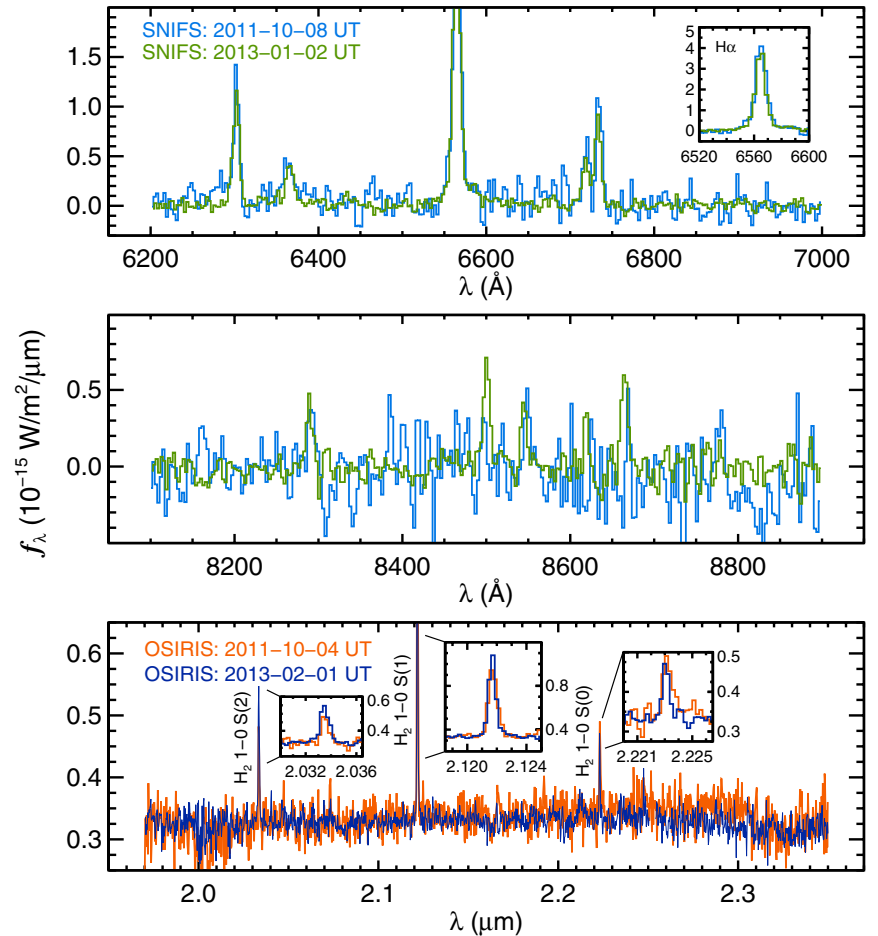

Figure 17. Comparison of our two epochs of flux-calibrated optical and NIR spectroscopy of FW Tau b. Most of the emission lines are constant over $\sim 1.5 \mathrm{yr}$, with only two showing evidence of variability at the $>3 \sigma$ level: $\mathrm{H}_{2} 1-0 S(2)$ and $[\mathrm{S} \mathrm{II}] \lambda 4067$. Insets show the relative intensities of the strongest lines in our data.

(A color version of this figure is available in the online journal.)

\subsubsection{Emission-line Variability}

Our multiple epochs of optical and NIR spectroscopy enable emission line strength comparisons over a span of $\sim 15$ months. Figure 17 shows our flux-calibrated spectra, and EW and line flux measurements are listed in Table 4. Most of the line fluxes agree. The only ones showing significant $(>3 \sigma)$ variability are [S II] $\lambda 4067$ and $\mathrm{H}_{2} 1-0 \mathrm{~S}(2)$, suggesting some changes in the shocked regions on $\sim 1$ yr timescales.

\subsubsection{Spatially Extended $\mathrm{H}_{2}$ Emission}

Extended $\mathrm{H}_{2}$ emission is commonly observed in the form of both bipolar jets and isolated outflows from young stars. NIR IFU observations in particular enable detailed spatial and kinematic studies of molecular hydrogen outflows (e.g., Beck et al. 2008; Davis et al. 2011; García López et al. 2013). To check for extended structure, we registered and coadded our nodded OSIRIS cubes for each emission line seen in our 2011 and 2013 data, subtracted continuum images adjacent to each line, and fit a 2D elliptical Gaussian to the source. The results of the fits are listed in Table 5. The FWHM of the $\mathrm{Pa} \beta, 1.257 \mu \mathrm{m}$ [Fe II], and $2.223 \mu \mathrm{m} \mathrm{H}_{2} 1-0 \mathrm{~S}(0)$ continuum-subtracted lines are consistent with the neighboring continuum emission, meaning the emission is not resolved and these lines originate from angular scales of $\lesssim 0$ '. 05 , or physical scales of $\lesssim 8$ AU at a distance of $\sim 145 \mathrm{pc}$.

On the other hand, the $2.122 \mu \mathrm{m} \mathrm{H}_{2} 1-0 S(1)$ and $2.034 \mu \mathrm{m}$ $\mathrm{H}_{2} 1-0 \mathrm{~S}(2)$ lines appear spatially extended compared to the continuum emission. This is verified by the FWHM measurements, which are about twice as large for the continuum-subtracted images compared to the continuum region for our 2011 data. Seeing and AO correction were substantially worse for our 2013 observations ( $\sim 150$ mas versus 100 mas in $K$ band), but these
Table 5

Fits to FW Tau b $\mathrm{H}_{2}$ Emission Line Images

\begin{tabular}{|c|c|c|c|c|c|}
\hline Line & Region & $\mathrm{FWHM}_{\text {maj }}$ & $a_{\max } / a_{\min }$ & P.A.inst & P.A.sky \\
\hline \multicolumn{6}{|c|}{2011 Oct 4 UT } \\
\hline \multirow[t]{3}{*}{$\mathrm{H}_{2} 1-0 S(2)$} & Emission line & 0.112 & 1.11 & 6 & 301 \\
\hline & Continuum & 0.105 & 1.25 & 23 & 318 \\
\hline & Cont-subtracted & 0.213 & 1.66 & 110 & 46 \\
\hline \multirow[t]{3}{*}{$\mathrm{H}_{2} 1-0 S(1)$} & Emission line & 0.134 & 1.09 & 138 & 73 \\
\hline & Continuum & 0.108 & 1.24 & 25 & 320 \\
\hline & Cont-subtracted & 0.195 & 1.38 & 124 & 60 \\
\hline \multirow[t]{3}{*}{$\mathrm{H}_{2} 1-0 S(0)$} & Emission line & 0.124 & 1.11 & 16 & 312 \\
\hline & Continuum & 0.111 & 1.12 & 175 & 110 \\
\hline & Cont-subtracted & 0.174 & 1.32 & 40 & 335 \\
\hline \multicolumn{6}{|c|}{2013 Feb 1 UT } \\
\hline \multirow[t]{3}{*}{$\mathrm{H}_{2} 1-0 S(2)$} & Emission line & 0.156 & 1.15 & 44 & 249 \\
\hline & Continuum & 0.149 & 1.20 & 72 & 277 \\
\hline & Cont-subtracted & 0.241 & 1.64 & 13 & 218 \\
\hline \multirow[t]{3}{*}{$\mathrm{H}_{2} 1-0 S(1)$} & Emission line & 0.188 & 1.09 & 175 & 21 \\
\hline & Continuum & 0.150 & 1.19 & 65 & 270 \\
\hline & Cont-subtracted & 0.248 & 1.28 & 168 & 13 \\
\hline \multirow[t]{3}{*}{$\mathrm{H}_{2} 1-0 S(0)$} & Emission line & 0.156 & 1.11 & 60 & 265 \\
\hline & Continuum & 0.161 & 1.26 & 71 & 276 \\
\hline & Cont-subtracted & 0.198 & 1.50 & 3 & 209 \\
\hline
\end{tabular}

data also reveal significantly extended emission. Figures 18 and 19 show images of the emission lines, adjacent continuum emission, and continuum-subtracted frames. The extended morphology appears asymmetric in the southwest direction. This is most evident for the 2011 1-0 S(1), 2011 1-0 S(2), and 2013 1-0 $S(2)$ lines, which have extensions at P.A.s of $240^{\circ},+226^{\circ}$, and $+218^{\circ}$, respectively. The extent of the lobe is $\sim 0$.' 1 from FW Tau b, or $\sim 15$ AU. Figure 20 shows cross sectional cuts of the $\mathrm{H}_{2} 1-0 S(1)$ and 1-0 $S(2)$ emission line images from Figures 18 and 19 along their semi-major axes. Compared to normalized adjacent continuum emission, the spatial extension and asymmetry are readily seen. The line emission is kinematically unresolved so no velocity information can be retrieved.

Dozens of MHOs have been discovered in Taurus (e.g., Beck et al. 2008; Davis et al. 2008; Khanzadyan et al. 2011). Since the outflow from FW Tau b is resolved, it has been assigned the name MHO 750 as the next addition to the online catalog of MHOs (Davis et al. 20109; C. Davis 2013, private communication), which follows IAU naming conventions. None of the known MHOs in Taurus are obviously associated with the FW Tau system.

\subsubsection{Physical Properties of the Emitting Gas}

The ratio of atomic and molecular emission lines spanning the optical to NIR can probe the physical conditions of the emitting region, including electron density, temperature, ionization fraction, and optical extinction (e.g., Hartigan et al. 1994). Several reliable line diagnostics have been established. The [S II] $\lambda 6716$ and $\lambda 6731$ lines have similar excitation energies, but different collisional strengths and transition probabilities, so their ratio can be used to determine $n_{e}$ (Osterbrock 1989). The critical densities for $\lambda 6716$ and $\lambda 6731$ are $\sim 1.5 \times 10^{3} \mathrm{~cm}^{-3}$ and $\sim 4 \times 10^{3} \mathrm{~cm}^{-3}$, respectively (Appenzeller \& Oestreicher 1988). At low densities $\left(n_{e} \lesssim 100 \mathrm{~cm}^{-3}\right)$, the line ratio is simply the ratio of statistical weights (1.5). At high densities $\left(n_{e} \gtrsim\right.$

\footnotetext{
9 http://www.astro.ljmu.ac.uk/MHCat/.
} 


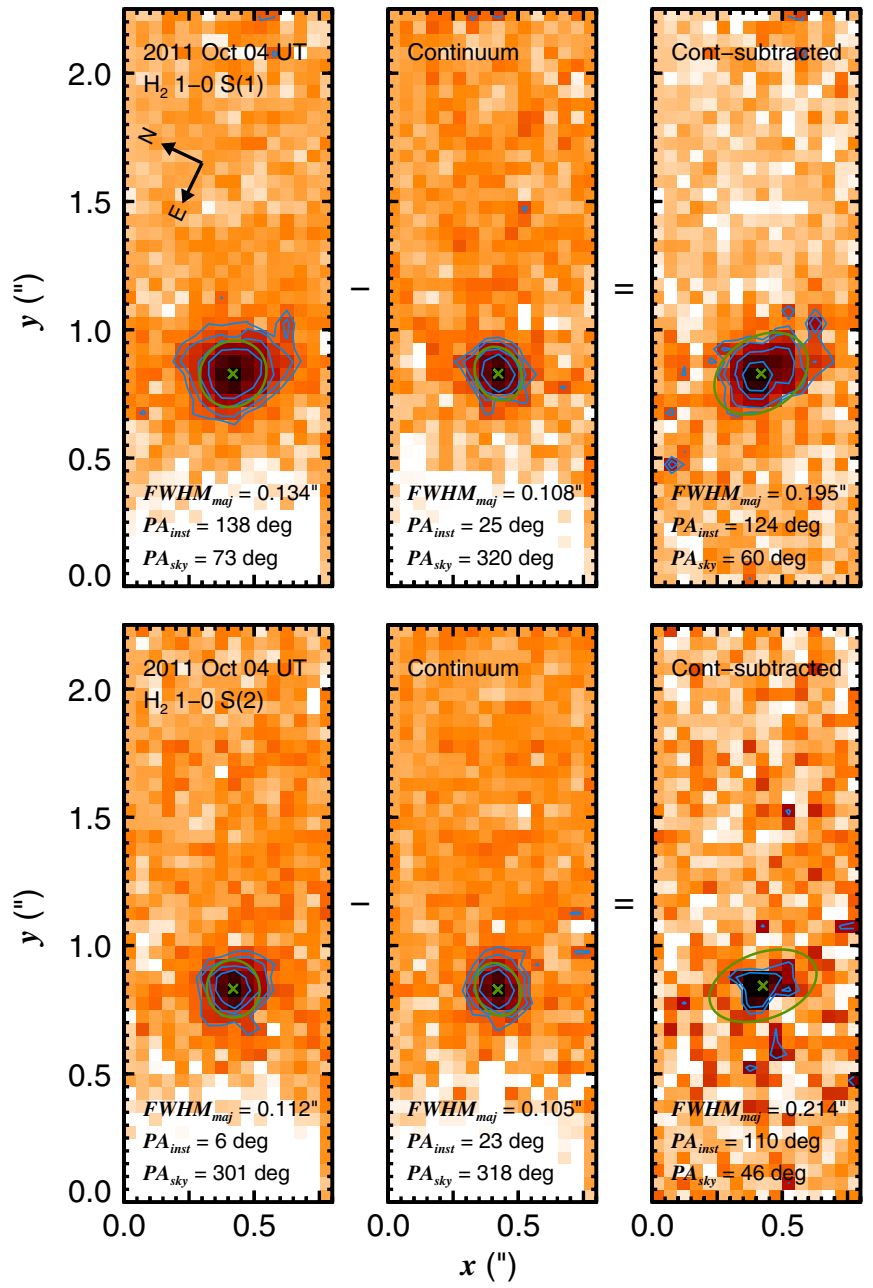

Figure 18. 2011 Keck/OSIRIS $\mathrm{H}_{2}$ images of FW Tau b. Each frame has been registered and coadded from our nodded observations. The top panels show $2.122 \mu \mathrm{m} \mathrm{H}_{2} 1-0 \mathrm{~S}(1)$ images of the emission line (left), continuum emission immediately adjacent to the line (center), and continuum-subtracted residuals (right). Blue contours represent $\{3 \sigma, 5 \sigma, 10 \sigma, 20 \sigma\}$ levels relative to the background rms. Green ellipses and crosses show the FWHM and centers from fitting a 2D elliptical Gaussian to the source. The FWHM of the major axis, instrumental position angle (P.A.), and sky P.A. are listed, and a compass rose is shown for orientation. The FWHM of the continuum-subtracted $\mathrm{H}_{2}$ 1-0 $S$ (1) line is about twice as large as the continuum image, indicating FW Tau b is marginally resolved along that axis. The extended emission appears asymmetric in the $+240^{\circ}$ direction. The bottom panels are identical for the $2.034 \mu \mathrm{m} \mathrm{H}_{2}$ 1-0 $S(2)$ emission line. It shows a similar slight, but significant, extension in the $+226^{\circ}$ direction. The morphology is similar to other $\mathrm{H}_{2}$ jets and probably originates from outflow material, although the structure is not kinematically resolved in our data.

(A color version of this figure is available in the online journal.)

$10^{4} \mathrm{~cm}^{-3}$ ) collisional de-excitation becomes significant and forbidden lines get weaker, producing a line ratio (0.44) equal to the statistical weights modulated by the transition probabilities. We measure values of $0.53 \pm 0.08$ and $0.7 \pm 0.3$ for our 2013 and 2011 data, respectively, implying an electron density of $\sim 6_{-3}^{+8} \times 10^{3} \mathrm{~cm}^{-3}$. (The large uncertainty in $n_{e}$ is caused by the steep dependence of $\lambda 6716 / \lambda 6716$ on $n_{e}$ near the high-density limit.) Raga et al. (1996) compute $n_{e}$ using the [S II] $\lambda 6716 /$ $\lambda 6731$ line ratio for $45 \mathrm{HH}$ condensations and find that most have $n_{e}$ below $5 \times 10^{3} \mathrm{~cm}^{-3}$. In comparison, the shocked gas in the vicinity of FW Tau $\mathrm{b}$ is near the high end for $\mathrm{HH}$ objects.

The $[\mathrm{Fe}$ II $] \lambda 7155 / \lambda 8617$ and $[\mathrm{Fe}$ II $] \lambda 12567 / \lambda 8617$ line ratios can also be used to probe electron density of the emitting
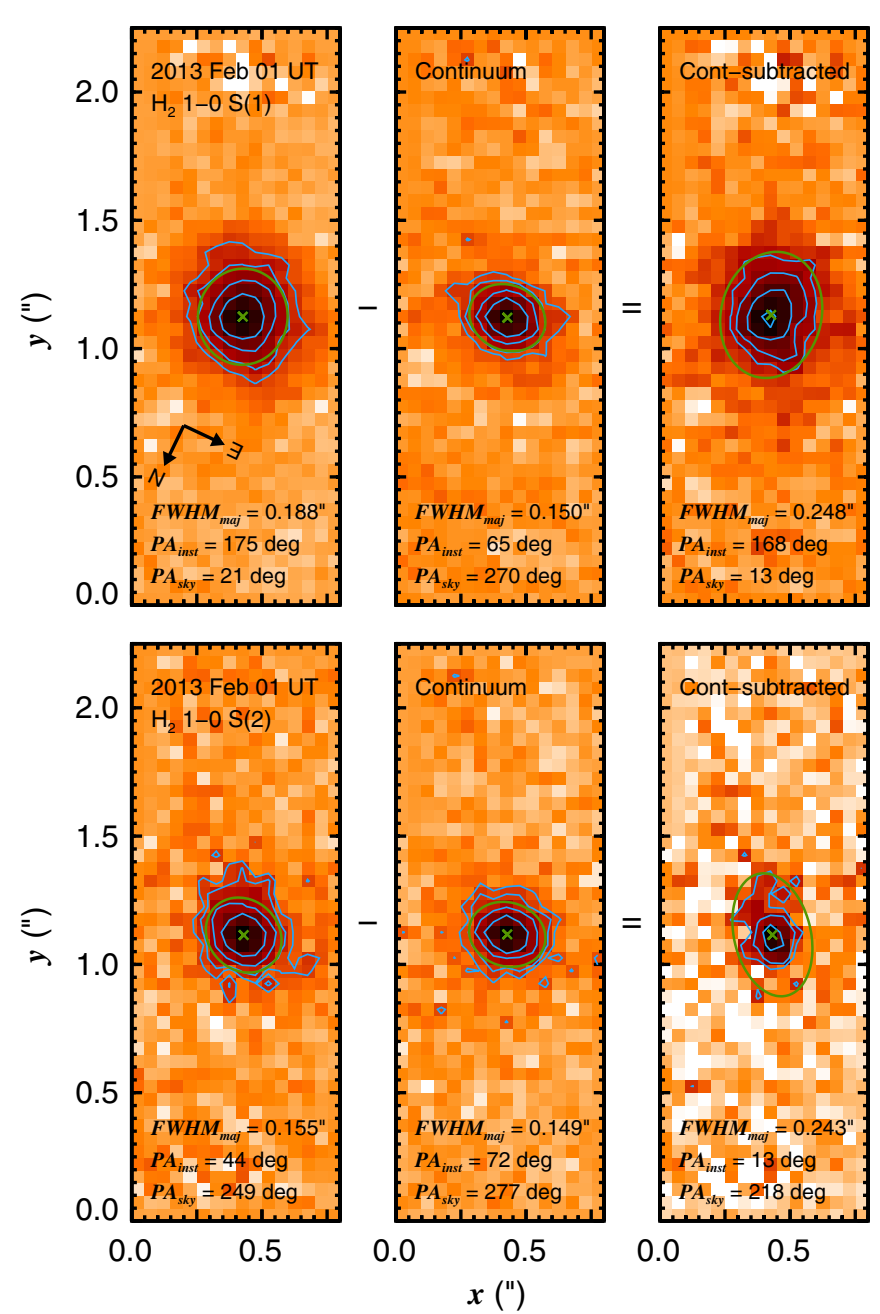

Figure 19. $\mathrm{H}_{2}$ images from our 2013 OSIRIS integral field observations of FW Tau b. The emission lines, contour plots, and Gaussian fits are identical to Figure 18. Seeing conditions and AO correction were substantially worse compared to our 2011 data, which is evident by the continuum FWHM measurements. Nevertheless, the continuum-subtracted $2.034 \mu \mathrm{m} \mathrm{H}_{2} 1-0 S(2)$ line show similar extension in the $+218^{\circ}$ direction and is broadly consistent with our 2011 observations. Note that the large difference in North orientation is a result of the different detector orientations (perpendicular here instead of parallel to the binary P.A.).

(A color version of this figure is available in the online journal.)

region. Unfortunately, the $\lambda 7155$ line is not detected, but our upper limit for the ratio $\lambda 7155 / \lambda 8617$ of $\lesssim 0.5$ implies electron densities of $\lesssim 10^{4} \mathrm{~cm}^{-3}$ (Bautista \& Pradhan 1996, 1998). This is consistent with our measurement from the [S II] $\lambda 6716 / \lambda 6731$ ratio. On the other hand, both $\lambda 12567$ and $\lambda 8617$ are detected, but the ratio we measure $(0.74 \pm 0.24)$ suggests higher values of $>10^{5} \mathrm{~cm}^{-3}$ (Bautista \& Pradhan 1998). This may indicate that multiple regions with different physical conditions are producing the $[\mathrm{Fe}$ II] lines.

\subsubsection{Accretion}

We use the empirical correlation between accretion luminosity $\left(L_{\text {acc }}\right)$ and $\mathrm{Pa} \beta$ luminosity $\left(L_{\mathrm{Pa} \beta}\right)$ from Natta et al. (2004, their Equation $(2))$ to derive a mass accretion rate $(\dot{M})$. A distance estimate of $145 \pm 10$ pc together with our measured $\mathrm{Pa} \beta$ line flux of $3.1 \pm 0.4 \mathrm{erg} \mathrm{s}^{-1} \mathrm{~cm}^{-2}$ yields a $\mathrm{Pa} \beta$ line luminosity of $\log \left(L_{\mathrm{Pa} \beta} / L_{\odot}\right)=-6.53 \pm 0.08 \mathrm{dex}$ and an accretion luminosity of $\log \left(L_{\text {acc }} / L_{\odot}\right)=-4.9 \pm 1.3$ dex. The accretion rate and 


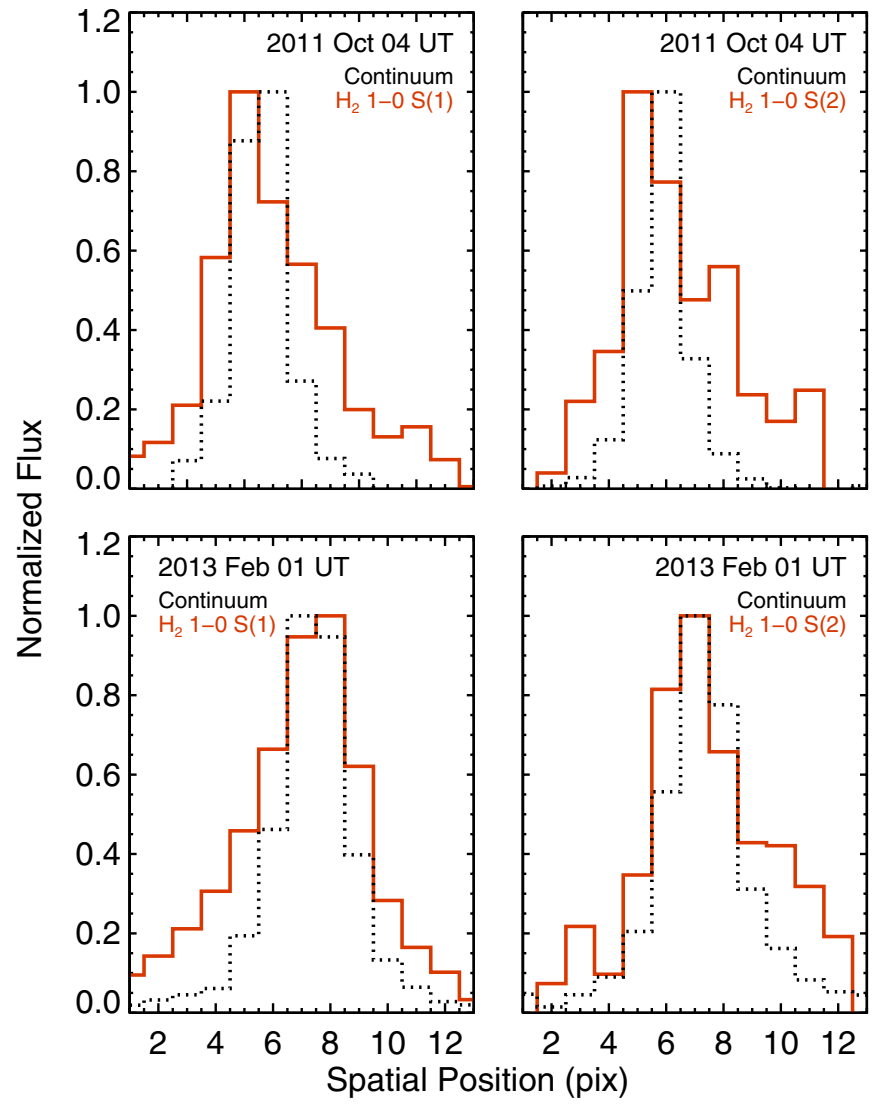

Figure 20. $\mathrm{H}_{2}$ 1-0 $S(1)$ and 1-0 $S(2)$ continuum-subtracted spatial line profiles (red) compared to adjacent continuum spatial profiles (dotted black). The panels show the spatial cross section of each emission line along its major axis of the continuum-subtracted frames from 2011 (Figures 18) and 2013 (Figure 19). In each case the normalized line profile appears extended compared to adjacent continuum, indicating resolved structure along that direction, most likely from outflow activity. Each cross section is oriented so that the SW direction of the cut is to the right. The OSIRIS plate scale is 50 mas spaxel $^{-1}$.

(A color version of this figure is available in the online journal.)

the accretion luminosity are related through $\dot{M}=L_{\text {acc }} R / G M$, where $R$ is object's radius and $M$ is its mass.

Since we do not have tight constraints on the mass of FW Tau $b$, we calculate accretion rates corresponding to the two physical scenarios: a veiled PMC, and an extincted low-mass star or brown dwarf with an edge-on disk. In the former case, we adopt the mass estimate of $10 \pm 4 M_{\text {Jup }}$ from Kraus et al. (2014), which corresponds to a radius of $0.21 \pm 0.05 R_{\odot}$ (Baraffe et al. 2003) and a mass accretion rate of $\log (\dot{M})=$ $-11.0 \pm 1.3 M_{\odot} \mathrm{yr}^{-1}$. This is consistent with accretion rate measurements of other substellar objects, which roughly follow an $\dot{M} \propto M^{2}$ relationship (Natta et al. 2004; Mohanty et al. 2005; Muzerolle et al. 2005; Herczeg \& Hillenbrand 2008). On the other hand, the higher mass range of $0.03-0.15 M_{\odot}$ corresponds to radii of $0.37-0.96 R_{\odot}$ (Baraffe et al. 1998) and a mass accretion rates of $-11.4 \pm 1.3 M_{\odot} \mathrm{yr}^{-1}$.

\section{DISCUSSION AND CONCLUSIONS}

There is strong evidence that companions near the deuteriumburning limit can form from multiple pathways. The HR 8799 planets offer the best support for non-hierarchical formation from a disk since initial constraints on their orbits are consistent with coplanarity (Esposito et al. 2012), and the planets are flanked by debris disks both inside and outside of their orbits (e.g., Su et al. 2009). On the other hand, the tail of the initial mass function (IMF) appears to continue down to at least $\sim 5-10 M_{\text {Jup }}$ (e.g., Luhman et al. 2009; Scholz et al. 2012; Alves de Oliveira et al. 2013; Liu et al. 2013), and PMCs have been found orbiting low-mass brown dwarfs with decidedly non-planetary mass ratios (Chauvin et al. 2004; Barrado y Navascués et al. 2007; Béjar et al. 2008; Todorov et al. 2010; Liu et al. 2012); together these suggest that cloud fragmentation and/or ejection can form objects that overlap with apparently bona fide planets (i.e., those born in a disk). These dual origins pose problems for interpreting discoveries from direct imaging planet searches, which are finding companions in this ambiguous 10-15 $M_{\text {Jup }}$ mass range (e.g., Chauvin et al. 2005; Itoh et al. 2005; Delorme et al. 2013; Bowler et al. 2013).

At the youngest ages, the disk properties of PMCs can provide constraints on their formation since dynamical scattering to wide orbits probably disrupts circum-planetary disks (Bowler et al. 2011; Bailey et al. 2013). Kraus et al. (2014) find that five out of seven of the PMCs in their sample have a redder $K-L^{\prime}$ color than field objects with the same spectral type, indicating that disks may be common to wide low-mass companions at very young ages. Strong $\mathrm{Pa} \beta$ emission from GSC 6214-210 $B$ unambiguously confirms the presence of an accretion disk (Bowler et al. 2011), and we show here that the same is true for FW Tau b, suggesting that it too probably formed in situ at several hundred AU. Hydrodynamic simulations by Shabram \& Boley (2013) show that planetary companions formed on wide orbits via disk instability have thick disks that are truncated at $\sim 1 / 3$ of the companion's Hill radius. GSC 6214-210 B and FW Tau b may be promising targets for follow-up observations to directly compare with these predictions.

The remarkable outflow activity we identified from FW Tau b provides some general clues about the formation and evolution of planetary-mass objects. Jets and outflows are common products of the star formation process, helping to shed disk angular momentum, regulate stellar mass, and contribute to turbulence in molecular clouds (e.g., Lada 1985; Richer et al. 2000; Quillen et al. 2005). In the substellar regime, disk accretion is commonly observed from young brown dwarfs, but evidence of outflows is much more rare because the mass accretion rate and mass loss are orders of magnitude smaller (Whelan et al. 2005; Whelan et al. 2009). One of the best-studied substellar outflows is the $\sim 24 M_{\text {Jup }}$ TWA member 2MASS J1207334-393254 A (Whelan et al. 2007, 2012), which until FW Tau b has been the lowest-mass jet-driving source known. The outflow itself appears collimated, bipolar, and altogether analogous to those from low-mass stars. To date, a handful of outflows from brown dwarfs have been detected in the optical through forbidden line emission (Joergens et al. 2012) and CO emission (Phan-Bao et al. 2008; Monin et al. 2013).

The outflow from FW Tau b makes it among only a handful of substellar objects showing such activity. If FW Tau b is confirmed to be planetary mass instead of a low-mass companion with an edge-on disk, it will be the first planetarymass object to drive an outflow. That would suggest that the same physical processes governing accretion, magnetic collimation, and angular momentum regulation in stars also operate at the bottom of the IMF. Distinguishing the veiled planetary-mass scenario from the higher-mass edge-on disk hypothesis should therefore be a chief goal for future studies of this system. One simple test is to search for weak photospheric features unique to $\mathrm{M}$ or $\mathrm{L}$ dwarfs in higher-S/N near-infrared spectroscopy; 
for example, the $2.21 \mu \mathrm{m} \mathrm{Na}$ I doublet appears in $\mathrm{M}$ dwarfs (though is weaker at lower gravities), but is absent in L dwarfs. Perhaps an outflow from such a low-mass companion should not be surprising since several free-floating planetary-mass objects are known to possess disks (e.g., Luhman et al. 2005), and theoretical arguments suggest that outflows might be natural byproducts of giant planet formation (Quillen \& Trilling 1998). Finally, we note that if the extended $\mathrm{H}_{2}$ emission from $\mathrm{FW}$ Tau $\mathrm{b}$ is confirmed to be a jet, it will be the first substellar molecular hydrogen outflow known.

We are grateful to the referee for prompt and useful feedback, Bo Reipurth and Lynne Hillenbrand for helpful comments on the properties of FW Tau b, Katelyn Allers for the low-gravity spectral templates used in this work, and Kimberly Aller and Will Best for carrying out some of the IRTF observations. It is also a pleasure to thank Joel Aycock, Randy Campbell, Heather Hershley, Jim Lyke, Julie Rivera, Hien Tran, Cindy Wilburn and the entire Keck staff for their support with the observations. B.P.B. and M.C.L. have been supported by NASA grant NNX11AC31G and NSF grant AST09-09222. Some of this work is based on observations obtained at the Gemini Observatory, which is operated by the Association of Universities for Research in Astronomy, Inc., under a cooperative agreement with the NSF on behalf of the Gemini partnership: the National Science Foundation (United States), the Science and Technology Facilities Council (United Kingdom), the National Research Council (Canada), CONICYT (Chile), the Australian Research Council (Australia), Ministério da Ciência, Tecnologia e Inovação (Brazil) and Ministerio de Ciencia, Tecnología e Innovación Productiva (Argentina) We utilized data products from the Two Micron All Sky Survey, which is a joint project of the University of Massachusetts and the Infrared Processing and Analysis Center/California Institute of Technology, funded by the National Aeronautics and Space Administration and the National Science Foundation. NASA's Astrophysics Data System Bibliographic Services together with the VizieR catalog access tool and SIMBAD database operated at CDS, Strasbourg, France, were invaluable resources for this work. Finally, mahalo nui loa to the kama'āina of Hawai' $i$ for their support of Keck and the Mauna Kea observatories. We are grateful to conduct observations from this mountain.

Facilities: Keck:II (OSIRIS, NIRC2), Keck:I (OSIRIS), Gemini:Gillett (NIFS), IRTF (SpeX), UH:2.2m (SNIFS)

\section{REFERENCES}

Aldering, G., Antilogus, P., Bailey, S., et al. 2006, ApJ, 650, 510 Alibert, Y., Mordasini, C., Benz, W., \& Winisdoerffer, C. 2005, A\&A, 434, 343 Aller, K. M., Kraus, A. L., Liu, M. C., et al. 2013, ApJ, 773, 63 Allers, K. N., Jaffe, D. T., Luhman, K. L., et al. 2007, ApJ, 657, 511 Allers, K. N., \& Liu, M. C. 2013, ApJ, 772, 79

Allers, K. N., Liu, M. C., Shkolnik, E., et al. 2009, ApJ, 697, 824 Alves de Oliveira, C., Moraux, E., Bouvier, J., et al. 2013, A\&A, 549, 123

Appenzeller, I., \& Oestreicher, R. 1988, AJ, 95, 45

Aspin, C., Beck, T. L., \& Reipurth, B. 2008, AJ, 135, 423

Bailey, V., Hinz, P. M., Currie, T., et al. 2013, ApJ, 767, 31

Bailey, V., Meshkat, T., Reiter, M., et al. 2014, ApJL, 780, L4

Baraffe, I., Chabrier, G., Allard, F., \& Hauschildt, P. H. 1998, A\&A, 337, 403

Baraffe, I., Chabrier, G., Barman, T. S., Allard, F., \& Hauschildt, P. H. 2003, A\&A, 402, 701

Barman, T. S., Macintosh, B., Konopacky, Q. M., \& Marois, C. 2011, ApJ, 733,65

Barrado y Navascués, D., Bayo, A., Morales-Calderón, M., et al. 2007, A\&A, 468, L5

Basri, G., \& Batalha, C. 1990, ApJ, 363, 654

Basri, G., \& Bertout, C. 1989, ApJ, 341, 340
Batalha, C. C., \& Basri, G. 1993, ApJ, 412, 363

Bate, M. R. 2012, MNRAS, 419, 3115

Bate, M. R., Bonnell, I. A., \& Bromm, V. 2002, MNRAS, 332, L65

Bautista, M. A., \& Pradhan, A. K. 1996, A\&AS, 115, 551

Bautista, M. A., \& Pradhan, A. K. 1998, ApJ, 492, 650

Beck, T. L., McGregor, P. J., Takami, M., \& Pyo, T.-S. 2008, ApJ, 676, 472

Béjar, V. J. S., Osorio, M. R. Z., Pérez-Garrido, A., et al. 2008, ApJL, 673, L185

Berthoud, M. G., Keller, L. D., Herter, T. L., Richter, M. J., \& Whelan, D. G. 2007, ApJ, 660, 461

Beuzit, J.-L., Ségransan, D., Forveille, T., et al. 2004, A\&A, 425, 997

Biller, B., Allers, K., Liu, M., Close, L. M., \& Dupuy, T. 2011, ApJ, 730, 39

Biller, B. A., Liu, M. C., Wahhaj, Z., et al. 2013, ApJ, 777, 160

Black, J. H., \& Dishoeck, E. F. V. 1987, ApJ, 322, 412

Bochanski, J. J., West, A. A., Hawley, S. L., \& Covey, K. R. 2007, AJ, 133, 531

Bonnefoy, M., Chauvin, G., Lagrange, A. M., et al. 2013, arXiv:1306.3709

Bonnefoy, M., Chauvin, G., Rojo, P., et al. 2010, A\&A, 512, A52

Boss, A. P. 1997, Sci, 276, 1836

Boss, A. P. 2006, ApJL, 637, L137

Boss, A. P. 2011, ApJ, 731, 74

Bouvier, J., \& Appenzeller, I. 1992, A\&AS, 92, 481

Bowler, B. P., Liu, M. C., \& Cushing, M. C. 2009, ApJ, 706, 1114

Bowler, B. P., Liu, M. C., Dupuy, T. J., \& Cushing, M. C. 2010, ApJ, 723, 850

Bowler, B. P., Liu, M. C., Kraus, A. L., Mann, A. W., \& Ireland, M. J. 2011, ApJ, 743,148

Bowler, B. P., Liu, M. C., Shkolnik, E. L., \& Dupuy, T. J. 2013, ApJ, 774, 55 Briceño, C., Hartmann, L., Stauffer, J., \& Martín, E. 1998, AJ, 115, 2074

Burgasser, A. J., Simcoe, R. A., Bochanski, J. J., et al. 2010, ApJ, 725, 1405

Burningham, B., Leggett, S. K., Homeier, D., et al. 2011, MNRAS, 414, 3590

Burrows, A., Marley, M., Hubbard, W. B., et al. 1997, ApJ, 491, 856

Busà, I., Cuadrado, R. A., Terranegra, L., Andretta, V., \& Gomez, M. T. 2007, A\&A, 466, 1089

Calvet, N., \& Gullbring, E. 1998, ApJ, 509, 802

Calvet, N., Hartmann, L., \& Kenyon, S. J. 1991, ApJ, 383, 752

Carpenter, J. M., Hillenbrand, L. A., \& Skrutskie, M. F. 2001, AJ, 121, 3160

Carr, J. S., Tokunaga, A. T., Najita, J., Shu, F. H., \& Glassgold, A. E. 1993, ApJL, 411, L37

Casali, M. M., \& Eiroa, C. 1996, A\&A, 306, 427

Chauvin, G., Lagrange, A.-M., Dumas, C., et al. 2004, A\&A, 425, L29

Chauvin, G., Lagrange, A.-M., Zuckerman, B., et al. 2005, A\&A, 438, L29

Chauvin, G., Menard, F., Fusco, T., et al. 2002, A\&A, 394, 949

Connelley, M. S., \& Greene, T. P. 2010, AJ, 140, 1214

Covey, K. R., Hillenbrand, L. A., Miller, A. A., et al. 2011, AJ, 141, 40

Cruz, K. L., Kirkpatrick, J. D., \& Burgasser, A. J. 2009, AJ, 137, 3345

Currie, T., Daemgen, S., Debes, J., et al. 2014, ApJL, 780, L30

Cushing, M. C., Kirkpatrick, J. D., Gelino, C. R., et al. 2011, ApJ, 743, 50

Cushing, M. C., Rayner, J. T., \& Vacca, W. D. 2005, ApJ, 623, 1115

Cushing, M. C., Vacca, W. D., \& Rayner, J. T. 2004, PASP, 116, 362

Davenport, J. R. A., Becker, A. C., Kowalski, A. F., et al. 2012, ApJ, 748, 58

Davis, C. J., Cervantes, B., Nisini, B., et al. 2011, A\&A, 528, A3

Davis, C. J., Gell, R., Khanzadyan, T., Smith, M. D., \& Jenness, T. 2010, A\&A, 511, A24

Davis, C. J., Scholz, P., Lucas, P., Smith, M. D., \& Adamson, A. 2008, MNRAS, 387,954

Davis, C. J., Whelan, E., Ray, T. P., \& Chrysostomou, A. 2003, A\&A, 397, 693

Delorme, P., Gagné, J., Girard, J. H., et al. 2013, A\&A, 553, L5

Dodson-Robinson, S. E., Veras, D., Ford, E. B., \& Beichman, C. A. 2009, ApJ, 707, 79

Duchêne, G., McCabe, C., Pinte, C., et al. 2010, ApJ, 712, 112

Eiroa, C., Oudmaijer, R. D., Davies, J. K., et al. 2002, A\&A, 384, 1038

Esposito, S., Mesa, D., Skemer, A., et al. 2012, A\&A, 549, A52

Fernández, M., \& Comerón, F. 2001, A\&A, 380, 264

Fitzpatrick, E. L. 1999, PASP, 111, 63

Folha, D. F. M., \& Emerson, J. P. 1999, A\&A, 352, 517

Garatti, A. C. O., Giannini, T., Nisini, B., \& Lorenzetti, D. 2006, A\&A, 449, 1077

García López, R., Caratti o Garatti, A., Weigelt, G., Nisini, B., \& Antoniucci, S. 2013, A\&A, 552, L2

Gautier, T. N., Fink, U., Larson, H. P., \& Treffers, R. R. 1976, ApJL, 207, L129

Giannini, T., Mccoey, C., Garatti, A. C. O., et al. 2004, A\&A, 419, 999

Giannini, T., Nisini, B., Garatti, A. C. O., \& Lorenzetti, D. 2002, ApJL, 570, L33

Goldman, B., Marsat, S., Henning, T., Clemens, C., \& Greiner, J. 2010, MNRAS, 405,1140

Golimowski, D. A., Henry, T. J., Krist, J. E., et al. 2004, AJ, 128, 1733

Gorlova, N. I., Meyer, M. R., Rieke, G. H., \& Liebert, J. 2003, ApJ, 593, 1074

Goulding, N. T., Barnes, J. R., Pinfield, D. J., et al. 2012, MNRAS, 427, 3358

Gredel, R. 1994, A\&A, 292, 580 
Greene, T. P., \& Lada, C. J. 1996, AJ, 112, 2184

Hamann, F., \& Persson, S. E. 1989, ApJ, 339, 1078

Hartigan, P., Edwards, S., \& Pierson, R. 2004, ApJ, 609, 261

Hartigan, P., Hartmann, L., Kenyon, S. J., Strom, S. E., \& Skrutskie, M. F. 1990, ApJL, 354, L25

Hartigan, P., Morse, J. A., \& Raymond, J. 1994, ApJ, 436, 125

Herbig, G. H., \& Vacca, W. D. 2008, AJ, 136, 1995

Herczeg, G. J., Cruz, K. L., \& Hillenbrand, L. A. 2009, ApJ, 696, 1589

Herczeg, G. J., \& Hillenbrand, L. A. 2008, ApJ, 681, 594

Herriot, G., Morris, S., Roberts, S., et al. 1998, Proc. SPIE, 3353, 488

Hillenbrand, L. A., Miller, A. A., Covey, K. R., et al. 2013, AJ, 145, 59

Hollenbach, D., \& McKee, C. F. 1989, ApJ, 342, 306

Huélamo, N., Bovy, H., Pinte, C., et al. 2010, A\&A, 523, A42

Ireland, M. J., Kraus, A., Martinache, F., Law, N., \& Hillenbrand, L. A. 2011, ApJ, 726, 113

Itoh, Y., Hayashi, M., Tamura, M., et al. 2005, ApJ, 620, 984

Jayawardhana, R., Luhman, K. L., D’Alessio, P., \& Stauffer, J. R. 2002, ApJL, 571, L51

Joergens, V., Pohl, A., Sicilia-Aguilar, A., \& Henning, T. 2012, A\&A, 543, A 151

Johns-Krull, C. M., \& Valenti, J. A. 2001, ApJ, 561, 1060

Khanzadyan, T., Movsessian, T. A., Davis, C. J., et al. 2011, MNRAS, 418, 1994

Kirkpatrick, J. D., Barman, T. S., Burgasser, A. J., et al. 2006, ApJ, 639, 1120

Kirkpatrick, J. D., Cushing, M. C., Gelino, C. R., et al. 2011, ApJSS, 197, 19

Kirkpatrick, J. D., Gelino, C. R., Cushing, M. C., et al. 2012, ApJ, 753, 156

Kirkpatrick, J. D., Looper, D. L., Burgasser, A. J., et al. 2010, ApJS, 190, 100

Konopacky, Q. M., Barman, T. S., Macintosh, B. A., \& Marois, C. 2013, Sci, 339, 1398

Kramida, A., Ralchenko, Y., Reader, J., \& Team, N. A. 2012, NIST Atomic Spectral Database (version 5.0, Online) (Gaithersburg, MD: National Institute of Standards and Technology)

Kratter, K. M., Murray-Clay, R. A., \& Youdin, A. N. 2010, ApJ, 710, 1375

Kraus, A. L., \& Ireland, M. J. 2012, ApJ, 745, 5

Kraus, A. L., Ireland, M. J., Cieza, L. A., et al. 2014, ApJ, 781, 20

Kraus, A. L., White, R. J., \& Hillenbrand, L. A. 2006, ApJ, 649, 306

Kuzuhara, M., Tamura, M., Ishii, M., et al. 2011, AJ, 141, 119

Kuzuhara, M., Tamura, M., Kudo, T., et al. 2013, ApJ, 774, 11

Lada, C. J. 1985, ARA\&A, 23, 267

Lafrenière, D., Jayawardhana, R., \& van Kerkwijk, M. H. 2008, ApJL, 689, L153

Lafrenière, D., Jayawardhana, R., \& van Kerkwijk, M. H. 2010, ApJ, 719, 497

Lagrange, A.-M., Bonnefoy, M., Chauvin, G., et al. 2010, Sci, 329, 57

Lantz, B., Aldering, G., Antilogus, P., et al. 2004, Proc. SPIE, 5249, 146

Larkin, J., Barczys, M., Krabbe, A., et al. 2006, Proc. SPIE, 6269, 42

Lépine, S., Hilton, E. J., Mann, A. W., et al. 2013, AJ, 145, 102

Lepp, S., \& McCray, R. 1983, ApJ, 269, 560

Liu, M. C., Delorme, P., Dupuy, T. J., et al. 2011, ApJ, 740, 108

Liu, M. C., Dupuy, T. J., Bowler, B. P., Leggett, S. K., \& Best, W. M. J. 2012, ApJ, 758,57

Liu, M. C., Dupuy, T. J., \& Ireland, M. J. 2008, ApJ, 689, 436

Liu, M. C., Magnier, E. A., Deacon, N. R., et al. 2013, ApJL, 777, L20

Liu, M. C., Najita, J., \& Tokunaga, A. T. 2003, ApJ, 585, 372

Lodieu, N., Dobbie, P. D., \& Hambly, N. C. 2011, A\&A, 527, A24

Lodieu, N., Hambly, N. C., Jameson, R. F., \& Hodgkin, S. T. 2008, MNRAS, 383, 1385

Looper, D. L., Bochanski, J. J., Burgasser, A. J., et al. 2010, AJ, 140, 1486

Lord, S. D. 1992, NASA Technical Memorandum 103957

Lucas, P. W., Roche, P. F., Allard, F., \& Hauschildt, P. H. 2001, MNRAS, 326, 695

Luhman, K. L. 1999, ApJ, 525, 466

Luhman, K. L. 2004a, ApJ, 602, 816

Luhman, K. L. 2004b, ApJ, 617, 1216

Luhman, K. L., Adame, L., D’Alessio, P., et al. 2005, ApJL, 635, L93

Luhman, K. L., Adame, L., D’Alessio, P., et al. 2007, ApJ, 666, 1219

Luhman, K. L., Briceño, C., Stauffer, J. R., et al. 2003a, ApJ, 590, 348

Luhman, K. L., Burgasser, A. J., \& Bochanski, J. J. 2011, ApJL, 730, L9

Luhman, K. L., Burgasser, A. J., Labbé, I., et al. 2012, ApJ, 744, 135

Luhman, K. L., Mamajek, E. E., Allen, P. R., \& Cruz, K. L. 2009, ApJ, 703, 399

Luhman, K. L., Peterson, D. E., \& Megeath, S. T. 2004, ApJ, 617, 565

Luhman, K. L., \& Rieke, G. H. 1998, ApJ, 497, 354

Luhman, K. L., \& Rieke, G. H. 1999, ApJ, 525, 440

Luhman, K. L., Stauffer, J. R., Muench, A. A., et al. 2003b, ApJ, 593, 1093

Luhman, K. L., Wilson, J. C., Brandner, W., et al. 2006, ApJ, 649, 894

Maloney, P. R., Hollenbach, D. J., \& Tielens, A. G. G. M. 1996, ApJ, 466, 561

Mann, A. W., Gaidos, E., \& Ansdell, M. 2013, ApJ, 779, 188

Mann, A. W., Gaidos, E., Lépine, S., \& Hilton, E. J. 2012, ApJ, 753, 90
Markwardt, C. B. 2009, in ASP Conf. Ser. 411, Astronomical Data Analysis Software and Systems XVIII, ed. D. A. Bohlender, D. Durand, \& P. Dowler (San Francisco, CA: ASP), 251

Marley, M. S., Fortney, J. J., Hubickyj, O., Bodenheimer, P., \& Lissauer, J. J. 2007, ApJ, 655, 541

Marois, C., Macintosh, B., Barman, T., et al. 2008, Sci, 322, 1348

Marois, C., Zuckerman, B., Konopacky, Q. M., Macintosh, B., \& Barman, T. 2010, Natur, 468, 1080

Marsh, K. A., Plavchan, P., Kirkpatrick, J. D., et al. 2010, ApJ, 719, 550

Mayer, L. 2002, Sci, 298, 1756

Mayne, N. J., \& Harries, T. J. 2010, MNRAS, 409, 1307

McClure, M. K., Calvet, N., Espaillat, C., et al. 2013, ApJ, 769, 73

McDonald, I., Zijlstra, A. A., \& Boyer, M. L. 2012, MNRAS, 427, 343

McGovern, M. R., Kirkpatrick, J. D., McLean, I. S., et al. 2004, ApJ, 600, 1020

McGregor, P. J., Hart, J., Conroy, P. G., et al. 2003, Proc. SPIE, 4841, 1581

McGregor, P. J., Persson, S. E., \& Cohen, J. G. 1984, ApJ, 286, 609

Moffat, A. F. J. 1969, A\&A, 3, 455

Mohanty, S., Jayawardhana, R., \& Basri, G. 2005, ApJ, 626, 498

Monin, J.-L., \& Bouvier, J. 2000, A\&A, 356, L75

Monin, J.-L., Whelan, E. T., Lefloch, B., Dougados, C., \& Alves de Oliveira, C. 2013, A\&A, 551, L1

Muench, A. A., Alves, J., Lada, C. J., \& Lada, E. A. 2001, ApJ, 558, L51

Muzerolle, J., Hartmann, L., \& Calvet, N. 1998, AJ, 116, 455

Muzerolle, J., Luhman, K. L., Briceño, C., Hartmann, L., \& Calvet, N. 2005, ApJ, 625, 906

Najita, J., Carr, J. S., Glassgold, A. E., Shu, F. H., \& Tokunaga, A. T. 1996, ApJ, 462,919

Natta, A., Testi, L., Muzerolle, J., et al. 2004, A\&A, 424, 603

Nielsen, E. L., \& Close, L. M. 2010, ApJ, 717, 878

Nisini, B., Bacciotti, F., Giannini, T., et al. 2005, A\&A, 441, 159

Nisini, B., Garatti, A. C. O., Giannini, T., \& Lorenzetti, D. 2002, A\&A, 393,1035

Oppenheimer, B. R., Baranec, C., Beichman, C., et al. 2013, ApJ, 768, 24

Osterbrock, D. E. 1989, Astrophysics of Gaseous Nebulae and Active Galactic Nuclei (Mill Valley, CA: University Science Books)

Patience, J., King, R. R., Rosa, R. J. D., et al. 2012, A\&A, 540, A85

Pecaut, M. J., Mamajek, E. E., \& Bubar, E. J. 2012, ApJ, 746, 154

Perryman, M. A. C., Lindegren, L., Kovalevsky, J., et al. 1997, A\&A, 323, L49

Phan-Bao, N., Riaz, B., Lee, C.-F., et al. 2008, ApJL, 689, L141

Pickles, A. 1998, PASP, 110, 863

Pollack, J. B., Hubickyj, O., Bodenheimer, P., et al. 1996, Icar, 124, 62

Preibisch, T., Guenther, E., Zinnecker, H., et al. 1998, A\&A, 333, 619

Press, W., Teukolsky, S. A., Vetterling, W. T., \& Flannery, B. P. 2007, Numerical Recipes: The Art of Scientific Computing (3rd ed.; Cambridge: Cambridge Univ. Press)

Quillen, A. C., Thorndike, S. L., Cunningham, A., et al. 2005, ApJ, 632, 941

Quillen, A. C., \& Trilling, D. E. 1998, ApJ, 508, 707

Raga, A. C., Böhm, K.-H., \& Cantó, J. 1996, RMxAA, 32, 161

Rameau, J., Chauvin, G., Lagrange, A.-M., et al. 2013, ApJ, 779, L26

Ratzka, T., Köhler, R., \& Leinert, C. 2005, A\&A, 437, 611

Rayner, J. T., Cushing, M. C., \& Vacca, W. D. 2009, ApJSS, 185, 289

Rebull, L. M., Padgett, D. L., McCabe, C.-E., et al. 2010, ApJSS, 186, 259

Reid, I. N., Hawley, S. L., \& Gizis, J. E. 1995, AJ, 110, 1838

Reipurth, B. 1999, A Genergal Catalog of Herbig-Haro Objects, 2nd ed., yCat, 5104 (http://cdsarc.u-strasbg.fr/viz-bin/Cat?V/104)

Reipurth, B., \& Aspin, C. 1997, AJ, 114, 2700

Reipurth, B., \& Bally, J. 2001, ARA\&A, 39, 403

Reipurth, B., Bally, J., Graham, J. A., Lane, A. P., \& Zealey, W. J. 1986, A\&A, 164,51

Rice, T. S., Wolk, S. J., \& Aspin, C. 2012, ApJ, 755, 65

Richer, J. S., Shepherd, D. S., Cabrit, S., Bachiller, R., \& Churchwell, E. 2000 in Protostars and Planets IV, ed. V. Mannings, A. P. Boss, \& S. S. Russell (Tucson, AZ: Univ. Arizona Press), 867

Rieke, G. H., \& Lebofsky, M. J. 1985, ApJ, 288, 618

Rodriguez, D. R., Zuckerman, B., Melis, C., \& Song, I. 2011, ApJL, 732, L29

Sartori, M. J., Lépine, J. R. D., \& Dias, W. S. 2003, A\&A, 404, 913

Scalzo, R. A., Aldering, G., Antilogus, P., et al. 2010, ApJ, 713, 1073

Scharf, C., \& Menou, K. 2009, ApJL, 693, L113

Scholz, A., \& Jayawardhana, R. 2006, ApJ, 638, 1056

Scholz, A., Jayawardhana, R., Muzic, K., et al. 2012, ApJ, 756, 24

Scholz, R.-D. 2010, A\&A, 515, A92

Schwartz, R. D. 1983, ARA\&A, 21, 209

Shabram, M., \& Boley, A. C. 2013, ApJ, 767, 63

Skrutskie, M. F., Cutri, R. M., Stiening, R., et al. 2006, AJ, 131, 1163

Skrutskie, M. F., Meyer, M. R., Whalen, D., \& Hamilton, C. 1996, AJ, 112,2168 
Slesnick, C. L., Hillenbrand, L. A., \& Carpenter, J. M. 2004, ApJ, 610, 1045

Song, I., Zuckerman, B., \& Bessell, M. S. 2003, ApJ, 599, 342

Spiegel, D. S., Burrows, A., \& Milsom, J. A. 2011, ApJ, 727, 57

Stapelfeldt, K. R., Menard, F., Watson, A. M., et al. 2003, ApJ, 589, 410

Su, K. Y. L., Rieke, G. H., Stapelfeldt, K. R., et al. 2009, ApJ, 705, 314

Takami, M., Chrysostomou, A., Ray, T. P., et al. 2006, ApJ, 641, 357

Thatte, N., Abuter, R., Tecza, M., et al. 2007, MNRAS, 378, 1229

Todorov, K., Luhman, K. L., \& Mcleod, K. K. 2010, ApJL, 714, L84

Tofflemire, B. M., Wisniewski, J. P., Kowalski, A. F., et al. 2011, AJ, 143, 12

Tokunaga, A. T. 2000, in Allen's Astrophysical Quantities, ed. A. N. Cox (4th ed.; New York, NY: AIP), 143

Vacca, W. D., Cushing, M. C., \& Rayner, J. T. 2003, PASP, 115, 389
Veras, D., Crepp, J. R., \& Ford, E. B. 2009, ApJ, 696, 1600

Wahhaj, Z., Cieza, L., Koerner, D. W., et al. 2010, ApJ, 724, 835

Watson, D. M., Kemper, F., Calvet, N., et al. 2004, ApJS, 154, 391

Whelan, E. T., Ray, T. P., Bacciotti, F., et al. 2005, Natur, 435, 652

Whelan, E. T., Ray, T. P., Comeron, F., Bacciotti, F., \& Kavanagh, P. J. 2012, ApJ, 761,120

Whelan, E. T., Ray, T. P., Podio, L., Bacciotti, F., \& Randich, S. 2009, ApJ, 706, 1054

Whelan, E. T., Ray, T. P., Randich, S., et al. 2007, ApJL, 659, L45

White, R. J., \& Ghez, A. M. 2001, ApJ, 556, 265

Yelda, S., Lu, J. R., Ghez, A. M., et al. 2010, ApJ, 725, 331

Zacharias, N., Finch, C. T., Girard, T. M., et al. 2013, AJ, 145, 44 